Article

\title{
Higher Derivative Gravity and Conformal Gravity from Bimetric and Partially Massless Bimetric Theory
}

\author{
Sayed Fawad Hassan ${ }^{1, *}$, Angnis Schmidt-May ${ }^{1,2}$ and Mikael von Strauss ${ }^{1,3}$ \\ ${ }^{1}$ Department of Physics \& The Oskar Klein Centre, Stockholm University, AlbaNova University \\ Centre, SE-106-91 Stockholm, Sweden; E-Mails: angniss@ phys.ethz.ch (A.S.-M.); \\ strauss@iap.fr (M.S.) \\ ${ }^{2}$ Institut für Theoretische Physik, Eidgenössische Technische Hochschule Zürich \\ Wolfgang-Pauli-Strasse 27, 8093 Zürich, Switzerland \\ ${ }^{3}$ UPMC-CNRS, UMR7095, Institut d'Astrophysique de Paris, GReCO, 98bis boulevard Arago, \\ F-75014 Paris, France
}

* Author to whom correspondence should be addressed; E-Mail: fawad@fysik.su.se.

Academic Editors: Kazuharu Bamba and Sergei D. Odintsov

Received: 12 May 2015 / Accepted: 9 July 2015 / Published: 20 July 2015

\begin{abstract}
In this paper, we establish the correspondence between ghost-free bimetric theory and a class of higher derivative gravity actions, including conformal gravity and new massive gravity. We also characterize the relation between the respective equations of motion and classical solutions. We illustrate that, in this framework, the spin-2 ghost of higher derivative gravity at the linear level is an artifact of the truncation to a four-derivative theory. The analysis also gives a relation between the proposed partially massless (PM) bimetric theory and conformal gravity, showing, in particular, the equivalence of their equations of motion at the four-derivative level. For the PM bimetric theory, this provides further evidence for the existence of an extra gauge symmetry and the associated loss of a propagating mode away from de Sitter backgrounds. The new symmetry is an extension of Weyl invariance, which may suggest the candidate PM bimetric theory as a possible ghost-free completion of conformal gravity.
\end{abstract}

Keywords: modified gravity; higher spin fields; conformal gravity 


\section{Introduction}

In this paper, we show the correspondence between the ghost-free bimetric theory [1,2] and higher derivative gravity, both of which have similar spectra, but only the bimetric case is ghost-free. In particular, this implies a close relation between conformal gravity and a specific bimetric theory that has been proposed [3,4] as the sought-after nonlinear partially massless (PM) theory. In this section, we start with a brief discussion of various theories considered in this paper, emphasizing the features that are of relevance here. We then present a summary of our results. In Section 2, we consider the correspondence for general bimetric parameters, and the ghost issue is discussed in Section 3. Section 4 is devoted to the PM bimetric theory and conformal gravity. Section 5 contains some discussions. A scalar field example is worked out in Appendix A, and some calculational details are relegated to Appendix B.

\subsection{A Review of the Different Theories Considered}

Below, we briefly review the relevant features of higher derivative gravity, conformal gravity, ghost-free bimetric theory and partially massless theories.

\subsubsection{Higher Derivative Gravity}

By this, we mean theories with more than two derivatives of the metric $g_{\mu \nu}$ that, at the four-derivative level, have the form,

$$
S_{(2)}^{\mathrm{HD}}[g]=m_{g}^{2} \int \mathrm{d}^{4} x \sqrt{g}\left[\Lambda+c_{R} R(g)-\frac{c_{R R}}{m^{2}}\left(R^{\mu \nu} R_{\mu \nu}-\frac{1}{3} R^{2}\right)\right] .
$$

This action propagates a massless spin- 2 state with two helicities, along with a massive spin- 2 state with five helicities, for a total of seven modes [5,6]. The massive spin-2 state of mass $m_{2}^{2}=m^{2} c_{R} / c_{R R}$ is a ghost; hence, it violates unitarity. Changing the coefficient of the $R^{2}$ term away from $\frac{1}{3}$ adds a massive scalar to the spectrum. Precisely for the form Equation (1), the scalar becomes infinitely massive and decouples. To Equation (1), one may add higher derivative terms suppressed by higher powers of $1 / \mathrm{m}^{2}$. The above action can be generalized to $d$ dimensions,

$$
S_{(2)}^{\mathrm{HD}}[g]=m_{g}^{d-2} \int \mathrm{d}^{d} x \sqrt{g}\left[\Lambda+c_{R} R(g)-\frac{c_{R R}}{m^{2}}\left(R^{\mu \nu} R_{\mu \nu}-\frac{d}{4(d-1)} R^{2}\right)\right] .
$$

For $d=3$ and $m_{g} c_{R}<0$, the action Equation (2) has been interpreted as a ghost-free theory of massive gravity, dubbed new massive gravity [7]. The choice $m_{g} c_{R}<0$ renders the massive spin-2 state healthy. However, the massless state, which is now a ghost, does not propagate in $d=3$, so the spectrum consists of a single massive spin-2 state. This construction is peculiar to $d=3$ and cannot cure the ghost problem for $d \geq 4$ [8,9]. For some work on higher derivative gravity, see [10-15].

\subsubsection{Conformal Gravity}

Dropping the first two terms in Equation (1), one obtains the action for conformal gravity [16],

$$
S^{\mathrm{CG}}[g]=-\frac{c_{R R} m_{g}^{2}}{m^{2}} \int \mathrm{d}^{4} x \sqrt{g}\left[R^{\mu \nu} R_{\mu \nu}-\frac{1}{3} R^{2}\right] .
$$


This is the square of the Weyl tensor (modulo the Euler density) and is invariant under Weyl scalings of the metric. It can also be constructed as the gauge theory of the conformal group [17]. The new Weyl invariance removes one of the seven propagating modes of Equation (1), so Equation (3) propagates only six modes $[18,19]$. In flat backgrounds, these appear as a healthy massless spin-2, a ghost-like massless spin-2 and massless spin-one [20]. In de Sitter (dS) backgrounds, the helicity 1 modes and one set of the helicity 2 modes become massive [21]. Conformal gravity appears in many different contexts and has played a role in several theoretical developments. For some recent work, a discussion and brief history, see [21-26].

\subsubsection{Ghost-Free Bimetric Theory}

This is formulated in terms of two metrics, $g_{\mu \nu}$ and $f_{\mu \nu}$, with an interaction potential described in Section 2.2. The bimetric action $S[g, f]$ is ghost-free and propagates seven modes [1,2] (see also [27,28]). For proportional metrics, $f_{\mu \nu}=c^{2} g_{\mu \nu}$, the classical solutions coincide with those of general relativity, and around such backgrounds, the seven modes split into massless and massive spin-2 fields [29]. Due to the presence of the massless field, this is not a theory of massive gravity. Rather, it describes gravity in the presence of a neutral spin-2 field in a completely dynamical setup. The bimetric spectrum is similar to that of higher derivative (HD) gravity Equation (1), except that neither of the spin-2 fields is a ghost. For some recent work within bimetric theory, see [30-44].

We refer to the ghost-free bimetric models as the Hassan-Rosen (HR) models, as distinct from the fixed background de Rham-Gabadadze-Tolley (dRGT) models [45,46] (for a recent review of massive gravity, see, e.g., [47]), to emphasize that they have very distinct dynamics and physics. The dRGT models, which propagate five nonlinear modes [48,49], are obtainable from the HR ones by dropping the kinetic term and the equation of motion for $f_{\mu \nu}$, which is then set to some fixed background metric, for instance $f_{\mu \nu}=\eta_{\mu \nu}$, as in the original dRGT setup [45,46]. This procedure cannot be implemented at the level of the bimetric action or the full bimetric equations of motion around generic backgrounds. It can only be implemented as a scaling limit on a class of bimetric solutions that survive in the limit. It is important to realize that when the massive gravity limit is taken for a certain class of solutions (with properly scaled parameters, so they remain regular), there also exists a large set of bimetric solutions that are singular in the massive gravity limit [50] (for example, bimetric theory has an interesting class of solutions that admit a general relativity limit without a vDVZ (van Dam-Veltman-Zakharov) discontinuity [51], while no such solutions exist in massive gravity). Therefore, the limit needs to be treated with care, and statements made in the massive gravity context do not automatically carry over to the bimetric theory, which has a richer solution space. In other words, the HR and dRGT setups not only have different field contents, but could also have very distinct dynamics and predictions. In particular, this difference is crucial for understanding that the recent analysis of acausality in massive gravity [52,53] and the arguments for the absence of partially massless theories in the fixed background setup [54,55] do not automatically apply to the HR theory. These works rely on the existence of a fixed reference metric $f_{\mu \nu}$ and, in the present form, manifestly break down in the fully dynamical bimetric setup. 


\subsubsection{Linear Partially Massless Theory and Beyond}

Partial masslessness was first observed in the Fierz-Pauli theory of a linear massive spin-2 field in a $\mathrm{dS}$ background. In $d=4$, it was found that when the mass and the cosmological constant satisfy the Higuchi bound, $m_{\mathrm{FP}}^{2}=\frac{2}{3} \Lambda$ [56], the massive spin-2 field has four propagating modes, instead of the usual five [57]. The reduction is due to a new gauge symmetry that emerges at the Higuchi bound. The resulting theory has been called partially massless. The obvious question is if there exists a nonlinear PM theory that goes beyond the Fierz-Pauli setup and that could even give a meaning to partial masslessness away from dS backgrounds (the PM phenomenon also arises in higher spin theories [58-60], but here, we consider only the spin-2 case).

A perturbative approach to finding such a theory has been to construct cubic vertices with PM gauge invariance in the linear Fierz-Pauli theory [60,61]. This has led to the insight that, in a two-derivative theory, cubic PM vertices exist only in three and four dimensions [59,62]. In higher derivative theories, such vertices can also be constructed in higher dimensions [59]. However, extending this method beyond cubic order becomes rather tedious.

Alternatively, a footnote in [21] observed that conformal gravity Equation (3) around dS backgrounds propagates a massless spin-2 field along with four massive modes that could be identified with the PM field. One of the two fields is now a ghost, depending on the overall sign of the action. The possibility of conformal gravity as a nonlinear PM theory was further investigated in [63]. The criterion was to look for a four-component PM field away from dS or Einstein backgrounds. (Linear PM modes exist in backgrounds specified by $R_{\mu \nu}=\Lambda g_{\mu \nu}$, i.e., in Einstein spacetimes and not just in dS spacetimes. From now on, this is assumed to be understood, even when not explicitly stated.) Such modes were not found, leading to the conclusion that conformal gravity is not a nonlinear PM theory, though it could still prove useful to the problem. From the bimetric point of view described below, the criterion in [63] is too restrictive, as it requires that the PM theory around any background looks similar to that around a dS background.

The main obstacle to a systematic construction of nonlinear PM theory is the relation to massive spin-2 fields that generically contain a Boulware-Deser ghost [64]. Hence, the search must focus on such ghost-free theories. The first of these is the dRGT model $[45,46]$, often described as massive gravity, though it also describes a massive spin-2 field $g_{\mu \nu}$ in a flat spacetime $\eta_{\mu \nu}$. The model is ghost-free nonlinearly [48], but it does not admit dS backgrounds [65] and, hence, does not accommodate even the linear PM theory. The generalizations of dRGT models to theories of a massive spin-2 field $g_{\mu \nu}$ in arbitrary curved backgrounds $f_{\mu \nu}$ [66] also exist and are free of the BD (Boulware-Deser) ghost [49,67]. These do admit dS backgrounds for $\bar{g}=\lambda^{2} f$ and can easily accommodate the known linear PM theory. A nonlinear PM candidate along these lines had been proposed in [68], but it was subsequently shown that this theory cannot accommodate an extra nonlinear gauge invariance [54,55].

\subsubsection{Partially Massless Bimetric Theory}

The HR bimetric theory [1] has dS solutions for $f_{\mu \nu}=c^{2} g_{\mu \nu}$, where, in general, $c^{2}$ is determined in terms of the parameters $\beta_{n}$ and $\alpha=m_{f} / m_{g}$ of the theory. In such backgrounds, the expressions for the cosmological constant and the Fierz-Pauli mass of the spin-2 fluctuations are known [4,29]. On 
imposing the Higuchi bound, one can easily recover the linear PM theory and its gauge symmetry. Then, as it turns out, a necessary condition for the consistency of the linear PM symmetry with the dynamical backgrounds is that the theory leaves $c^{2}$ undetermined [3,4]. This uniquely determines a specific bimetric theory as the candidate nonlinear PM theory that exists even away from dS or Einstein backgrounds.

Although the gauge symmetry away from dS backgrounds is not yet known, there are indications that it exists, in which case the theory will propagate six modes (instead of the seven for a generic HR model) even away from dS backgrounds. However, only around dS backgrounds, these can be decomposed as four components of the PM field and two helicities of a massless graviton [29]. Otherwise, the six modes will not have such simple decompositions in terms of mass eigenstates. Such a behavior is not unexpected from a field theoretical perspective due to the nonlinear interactions of fields with the same spin. Hence, our criterion for identifying a nonlinear generalization of the known PM theory is that it has six propagating modes (instead of the generic seven), irrespective of how they decompose. (In the absence of a nonlinearly identifiable four-component PM multiplet, one may object to labelling these as PM theories. We use the name at least for historical reasons, but more importantly, since these are seemingly the only possible nonlinear generalizations of the linear PM theories.)

One piece of evidence in favor of the bimetric PM proposal is that, in a two derivative setup, it correctly predicts nonlinear PM theories only in $d=3$ and $d=4$. However, on allowing for higher derivative terms, one obtains candidate PM theories, even in higher dimensions [4]. This is in accordance with the outcome of the direct construction of PM cubic vertices mentioned above (for some more arguments, see $[3,4]$ ). Further evidence for the existence of an extra symmetry in the PM bimetric theory will be provided below.

It should also be emphasized that, even if it eventually turns out that the bimetric setup cannot provide a nonlinear description of PM theory, in the sense that only six degrees of freedom are propagating, the candidate theory is still worth studying. Its special properties and, in particular, the connection to conformal gravity that will be established in this work provide a richer framework for studying partial masslessness, possibly within an extension of bimetric theory. It would then be feasible that, in this larger framework, the additional gauge symmetry does exist, while in bimetric theory, we only discover remnants of it.

\subsection{Summary of Results}

Our results are summarized below:

Correspondence between bimetric theory and HD gravity: Starting with the bimetric action $S[g, f]$ in terms of spin-2 fields $g_{\mu \nu}$ and $f_{\mu \nu}$, we determine $f_{\mu \nu}$ algebraically in terms of $g_{\mu \nu}$ and its curvatures $R_{\mu \nu}(g)$. In general, the solution $f_{\mu \nu}(g)$ is a perturbative expansion in powers of $R_{\mu \nu}(g) / m^{2}$, where $m^{2}$ sets the scale of the Fierz-Pauli mass in the bimetric theory. While this expression is valid for $R(g)<m^{2}$, in special cases, the series terminates and the solution is exact. Using this solution to eliminate $f_{\mu \nu}$ from the bimetric action, we obtain the action for higher derivative gravity as,

$$
S^{\mathrm{HD}}[g]=S[g, f(g)] .
$$

At the four-derivative level, this gives $S_{(2)}^{\mathrm{HD}}$ in Equation (1). We perform this calculation in $d$ dimensions, and hence, for $d=3$, we can obtain a higher derivative extension of the new massive 
gravity setup of [7] as dictated by the bimetric theory. (For another attempt to obtain new massive gravity from bimetric theory, involving certain scaling limits of the parameters, see [69]. In that approach, one introduces spin-2 ghosts already in bimetric theory by taking the wrong sign for the $f$-metric kinetic term. In the present paper, we keep the bimetric theory healthy and try to pin point the origin of the ghost in the higher-derivative theory.)

We also obtain a correspondence between the equations of motion in HD gravity and bimetric theory. These differ by an extra operator $(\delta f / \delta g)$. At the linearized level, if $\chi(x)$ solves the differential equation $(\delta f / \delta g) \chi=0$, then the solutions of the HD gravity equations have the form $g^{\mathrm{HD}}=g^{\mathrm{HD}}(x, \chi(x))$. The classical solutions in bimetric theory are related to these by,

$$
g^{\mathrm{BM}}(x)=g^{\mathrm{HD}}(x, \chi=0) .
$$

Thus, on setting $\chi=0$, say, through boundary conditions, the classical solutions in the two theories will coincide.

Truncation and ghost in HD gravity: The HD gravity action truncated to quadratic curvature terms, $S_{(2)}^{\mathrm{HD}}$, Equation (1), has seven propagating modes that, around appropriate backgrounds, decompose into a massless and a massive spin-2 field. This is similar to the bimetric spectrum, except for two important differences. The massive spin-2 field in $S_{(2)}^{\mathrm{HD}}$ is a ghost, and its mass differs from the value in the associated bimetric theory. We illustrate that, in the linear theory, these discrepancies are the artifacts of truncating HD gravity to a four-derivative theory. Resolving the ghost issue then also requires using appropriate source couplings, as dictated by the associated bimetric theory.

To make this explicit, in the Appendix, we consider two examples, the linearized bimetric theory and a very similar, but much simpler theory of two scalars fields. In both cases, one of the fields can be eliminated to explicitly obtain a higher derivative action for the remaining field, analogous to the untruncated $S^{\mathrm{HD}}$. This action contains appropriate modified source couplings inherited from the bi-field theory. To check for ghosts, we compute the vacuum persistence amplitude, which turns out to be exactly the same as the one computed in the associated bi-field theory. Hence, the untruncated HD theory is ghost free and has the right mass poles. However, one can see that if this theory is truncated to four-derivative terms, the massive field becomes a ghost, and its mass shifts away from the correct value.

Conformal gravity and the PM bimetric theory: For the specific bimetric theory that has been identified as the candidate nonlinear PM theory [3,4], the corresponding HD theory at the four-derivative level is conformal gravity Equation (3). What is more, in this case, the correspondence is closer to an equivalence: while in general, the truncated equations in HD gravity and bimetric theory are not the same, in the PM case, the lowest order bimetric equation is identical to the conformal gravity equation, expressed as the vanishing of the Bach tensor,

$$
B_{\mu \nu}(g)=0
$$

For the PM bimetric theory, the implication is that, in the limit $R(g)<<m^{2}$, it propagates six (rather than seven) modes even away from dS backgrounds. The new gauge symmetry that exists in the limit $R(g)<<m^{2}$, not only around dS backgrounds, but around any Bach flat background, corresponds to Weyl transformations of $g_{\mu \nu}$. This is a further indication that the full PM bimetric theory indeed has an 
extra gauge symmetry. Since the Bach equation is now derived from the bimetric action instead of the conformal gravity (CG) action, none of the six modes it propagates is a ghost.

Conversely, the above equivalence implies that the PM bimetric theory is a genuine extension of conformal gravity that resolves its ghost problem. In the process, the Weyl symmetry of CG is replaced by the PM symmetry of bimetric theory.

From the bimetric perspective, it is obvious that only around proportional dS backgrounds, the six modes split into a massless graviton and a four-component PM field. Away from dS backgrounds, the six modes do not admit such a split [29], and in particular, it is not possible to freeze gravity and retain only the PM field, as attempted in [63].

\section{Higher Curvature Gravity from Bimetric Theory}

Starting with the bimetric theory, we will now obtain the higher derivative gravity actions as an expansion in the inverse of the mass scale. We will explicitly compute quadratic curvature terms after first outlining the procedure and a brief review of the ghost-free bimetric theory.

\subsection{Outline of Obtaining Higher Derivative Gravity from Bimetric Theory}

The bimetric action $S[g, f]$ involves the two spin-2 fields $g_{\mu \nu}$ and $f_{\mu \nu}$ with a non-derivative interaction potential to be specified later. The corresponding equations of motion,

$$
\left.\frac{\delta S}{\delta g^{\mu \nu}}\right|_{f}=0,\left.\quad \frac{\delta S}{\delta f^{\mu \nu}}\right|_{g}=0,
$$

are coupled differential equations and include the sources $T_{\mu \nu}^{g}$ and $T_{\mu \nu}^{f}$. Eliminating one of the metrics, say $f_{\mu \nu}$, between the two equations leads to a higher derivative equation for $g_{\mu \nu}$. Of course, this is just a step in the direction of solving Equation (7), and the resulting higher derivative equation is completely within the framework of bimetric theory. However, as will be exhibited for a scalar field in the Appendix, it is not straightforward to derive this higher derivative equation directly from a local ghost-free action for $g_{\mu \nu}$ alone. Therefore, this manipulation will not lead to standard higher curvature gravity actions.

On the other hand, note that the $g_{\mu \nu}$ equations do not contain derivatives of $f_{\mu \nu}$. Hence, they can be solved algebraically to determine $f_{\mu \nu}$ in terms of $g_{\mu \nu}$ and its curvatures, at least perturbatively. This will yield $f=f\left(g, T^{g}\right)$. Using this to eliminate $f_{\mu \nu}$ in the bimetric action $S[g, f]$ then leads to a higher derivative action for $g_{\mu \nu}$, which precisely coincides with the standard class of higher curvature gravity actions. Before getting into the details, let us clarify the relation between the equations of motion in HD gravity and in bimetric theory.

Suppose one obtains $f=f(g)$ by solving either of the equations in Equation (7). This can be used to convert the bimetric action into a higher derivative action $S^{\prime}[g]=S[g, f(g)]$ for $g$. Varying $S^{\prime}$ with respect to $g$ gives,

$$
\frac{\delta S^{\prime}}{\delta g(x)}=\left.\frac{\delta S}{\delta g(x)}\right|_{f(g)}+\left.\int \mathrm{d}^{d} y \frac{\delta f(y)}{\delta g(x)} \frac{\delta S}{\delta f(y)}\right|_{g}=0 .
$$

The first term corresponds to the usual bimetric $g$-equation of motion Equation (8), while the second term corresponds to the bimetric $f$ equation of motion, multiplied by a Jacobian. Two cases arise depending on how $f_{\mu \nu}$ is determined. 
If $f_{\mu \nu}$ is a solution to the $f$-equation $\left.\frac{\delta S}{\delta f}\right|_{g}=0$, say as $f=f\left(g, T^{f}\right)$, then the second term vanishes, and Equation (8) implies exactly the bimetric $g$-equation. However, this requires solving a differential equation for $f_{\mu \nu}$ (which is not easy to solve in full generality), and the solution depends on the boundary conditions. Hence, in this case, the action $S^{\prime}$ is bi-local, and the resulting theory is completely equivalent to the bimetric theory. This approach is not the focus of our attention in the present paper.

Now, suppose that we instead use the bimetric $g$-equation, $\left.\frac{\delta S}{\delta g}\right|_{f}=0$, to algebraically determine $f=f\left(g, T^{g}\right)$. Since the solution is algebraic, $S^{\prime}[g]$ is a local higher derivative action for $g$ and coincides with $S^{\mathrm{HD}}[g]$, Equation (4). Later, we will see that, when expanded in powers of curvatures, it reproduces the standard higher derivative gravity action Equation (2) at the lowest orders. From Equation (8), the equation of motion becomes,

$$
\frac{\delta S^{\mathrm{HD}}}{\delta g(x)}=\left.\int \mathrm{d}^{d} y \frac{\delta f(y)}{\delta g(x)} \frac{\delta S}{\delta f(y)}\right|_{g}=0
$$

The Jacobian factor is in fact an operator, $\frac{\delta f(y)}{\delta g(x)}=\delta(x-y) \hat{\mathcal{O}}_{y}$, so the higher derivative $g$-equation of motion is,

$$
\hat{\mathcal{O}}\left(\left.\frac{\delta S[g, f]}{\delta f}\right|_{g}\right)=0
$$

where $f=f\left(g, T^{g}\right)$. This differs form the corresponding bimetric equation by the presence of the extra operator $\hat{\mathcal{O}}$. For functions $\chi$, such that $\hat{\mathcal{O}} \chi=0$, we get,

$$
\left.\frac{\delta S[g, f]}{\delta f}\right|_{g}=\chi(x)
$$

Thus, from the bimetric point of view, $\chi(x)$ appears as an unusual source term specified through boundary conditions. Let us denote a solution of this equation by $g^{\mathrm{HD}}(x, \chi(x))$. Thus, only for boundary conditions that give $\chi=0$, the solutions of the higher curvature action $S^{\mathrm{HD}}[g]$ coincide with the solutions of bimetric theory, $g^{\mathrm{BM}}(x)=g^{\mathrm{HD}}(x, \chi=0)$.

The difference between the bimetric and HD gravity equations, contained in $\hat{\mathcal{O}}$ is easy to isolate formally. However, in the truncated theory, $\hat{\mathcal{O}}$ mixes with $\delta S / \delta f$, and generically, the truncated equations are not equivalent (an exception being conformal gravity). In particular, the higher derivative theory, when truncated to four-derivative terms, will contain spin-2 ghosts. However, as the scalar field example in the Appendix illustrates, these are artifacts of the truncation and of using a naive source coupling instead of the one dictated by the parent bimetric theory. Since the truncation scale is given by the mass scale, the truncated theory is meaningful only at energies below the mass scale. Conversely, the bimetric theory seems to provide a ghost-free completion of quadratic curvature gravity.

\subsection{Review of Ghost-Free Bimetric Gravity}

Let us briefly review the relevant equations of HR bimetric theory [1]. More details can be found in $[29,66]$ and in the appendix of [4]. The bimetric action is,

$$
S[g, f]=m_{g}^{d-2} \int \mathrm{d}^{d} x\left(\sqrt{g} R(g)+\alpha^{d-2} \sqrt{f} R(f)-2 m^{2} \sum_{n=0}^{d} \beta_{n} e_{n}(S)\right),
$$


where $S$ stands for the square-root matrix $S^{\mu}{ }_{\nu}=\left(\sqrt{g^{-1} f}\right)^{\mu}{ }_{\nu}$ and $e_{n}(S)$ are the elementary symmetric polynomials of the eigenvalues of $S$. Starting with $e_{0}(S)=1$, they are constructed iteratively through,

$$
e_{n}(S)=-\frac{1}{n} \sum_{k=1}^{n}(-1)^{k} \operatorname{Tr}\left(S^{k}\right) e_{n-k}(S) .
$$

In $d$ dimensions, $e_{d}(S)=\operatorname{det} S$ and $e_{n}(S)=0$ for $n>d$. We will explicitly need,

$$
e_{1}(S)=\operatorname{Tr} S, \quad e_{2}(S)=\frac{1}{2}\left[(\operatorname{Tr} S)^{2}-\left(\operatorname{Tr} S^{2}\right)\right] .
$$

In Equation (12), $\alpha=m_{f} / m_{g}$ is the ratio of the Planck masses, and $\beta_{n}$ are $d+1$ dimensionless free parameters. $m^{2}$, which is degenerate with the overall scale of the $\beta_{n}$, sets the mass scale of the massive mode. The action retains its form under the interchange of $g$ and $f$ due to the properties of the $e_{n}(S)$ [1]. The equations of motion for $g_{\mu \nu}$ and $f_{\mu \nu}$ are,

$$
\begin{aligned}
& R_{\nu}^{\mu}(g)-\frac{1}{2} \delta_{\nu}^{\mu} R(g)+m^{2} \sum_{n=0}^{d-1}(-1)^{n} \beta_{n} \mathbb{Y}_{(n) \nu}^{\mu}(S)=m_{g}^{2-d} T_{\nu}^{g \mu} \\
& R_{\nu}^{\mu}(f)-\frac{1}{2} \delta_{\nu}^{\mu} R(f)+\frac{m^{2}}{\alpha^{d-2}} \sum_{n=1}^{d}(-1)^{n} \beta_{n} \mathbb{Y}_{(n) \nu}^{\mu}\left(S^{-1}\right)=m_{f}^{2-d} T_{\nu}^{f^{\mu}}
\end{aligned}
$$

where the matrices $\mathbb{Y}_{(n)}$ are given by (see, for example, [4]),

$$
\mathbb{Y}_{(n)}(S)=\sum_{k=0}^{n}(-1)^{k} e_{k}(S) S^{n-k}
$$

Around proportional backgrounds, $f_{\mu \nu}=c^{2} g_{\mu \nu}$, the degrees of freedom propagated by the action Equation (12) split into a massless and a massive spin-2 field. In the present conventions, the Fierz-Pauli mass is given by [29],

$$
m_{\mathrm{FP}}^{2}=m^{2}\left(\frac{1+(\alpha c)^{d-2}}{(\alpha c)^{d-2}}\right) \sum_{n=1}^{d-1}\left(\begin{array}{l}
d-2 \\
n-1
\end{array}\right) c^{n} \beta_{n} .
$$

In general, $c$ is determined in terms of $\beta_{n}$ and $\alpha$ through the equality of the cosmological constants, $\Lambda_{g}=\Lambda_{f}$. Furthermore, if $\Lambda_{g}=0$, then Equation (18) gives the mass around flat spacetime.

\subsection{The Algebraic Solutions for $S$ and $f$}

The $g$-equation, Equation (15), is algebraic in $S_{\nu}^{\mu}$ and, hence, in $f_{\mu \nu}$, so it can be used to determine $f_{\mu \nu}$ in terms of $g_{\mu \nu}$ its curvatures and $T_{\mu \nu}^{g}$. First, we consider the minimal $\beta_{1}$ model (with all $\beta_{n}$ for $n \geq 2$ vanishing) where the solution can be found exactly and then find a perturbative solution in powers of the curvatures for general $\beta_{n}$.

\subsubsection{Exact Solution in the $\beta_{1}$ Model:}

In the $\beta_{1}$ model (where $\beta_{2}=\cdots=\beta_{d-1}=0$ ), the $g_{\mu \nu}$ equation of motion becomes,

$$
G_{\nu}^{\mu}(g)+m^{2}\left[\beta_{0} \delta_{\nu}^{\mu}-\beta_{1}\left(S_{\nu}^{\mu}-\delta_{\nu}^{\mu} \operatorname{Tr} S\right)\right]=m_{g}^{2-d} T_{\nu}^{g \mu},
$$


where $G_{\mu \nu}=R_{\mu \nu}-\frac{1}{2} g_{\mu \nu} R$ is the Einstein tensor. The trace of this equation determines $\operatorname{Tr} S$. Then, for $T^{g \mu}{ }_{\nu}=0$, one finds the solution,

$$
S_{\nu}^{\mu}=-\frac{1}{d-1} \frac{\beta_{0}}{\beta_{1}} \delta_{\nu}^{\mu}+\frac{1}{m^{2} \beta_{1}} P_{\nu}^{\mu}
$$

Here, $P_{\nu}^{\mu}=g^{\mu \lambda} P_{\lambda \nu}$ is the Schouten tensor of $g_{\mu \nu}$ in $d$ dimensions (the conventionally normalized Schouten tensor is $\frac{1}{d-2} P_{\mu \nu}$, but the non-standard normalization of Equation (21) is more convenient for our purposes),

$$
P_{\mu \nu}=R_{\mu \nu}-\frac{1}{2(d-1)} g_{\mu \nu} R
$$

From $S$, one can easily find $f_{\mu \nu}=g_{\mu \lambda}\left(S^{2}\right)_{\nu}^{\lambda}$. The solution has the form of a power series in $P / m^{2}$ that terminates at the first order.

The solution for $S$ in the presence of matter sources is easily obtained from Equation (20) by the replacement $G_{\mu \nu} \rightarrow G_{\mu \nu}-m_{g}^{2-d} T_{\mu \nu}^{g}$, or equivalently by,

$$
\left.P_{\nu}^{\mu} \rightarrow P_{\nu}^{\mu}-m_{g}^{2-d}\left(T_{\nu}^{g \mu}-\frac{1}{d-1} T_{\lambda}^{g \lambda} \delta_{\nu}^{\mu}\right)\right)
$$

\subsubsection{Perturbative Solution for General $\beta_{n}$ :}

For general $\beta_{n}$ in Equation (15), finding an exact solution for $S=\sqrt{g^{-1} f}$ is not feasible. However, one can always find a perturbative solution as an expansion in $m^{-2}$. In the absence of matter sources, this corresponds to a derivative expansion in powers of $R_{\mu \nu}(g) / m^{2}$, or equivalently, in $P_{\mu \nu}(g) / m^{2}$. In order to find the perturbative solution, we make the general ansatz,

$$
\begin{aligned}
S_{\nu}^{\mu}=a \delta_{\nu}^{\mu} & +\frac{1}{m^{2}}\left(b_{1} P_{\nu}^{\mu}+b_{2} \operatorname{Tr} P \delta_{\nu}^{\mu}\right) \\
& +\frac{1}{m^{4}}\left(c_{1} P_{\nu}^{\mu 2}+c_{2} P_{\nu}^{\mu} \operatorname{Tr} P+c_{3} \operatorname{Tr}\left(P^{2}\right) \delta_{\nu}^{\mu}+c_{4}(\operatorname{Tr} P)^{2} \delta_{\nu}^{\mu}\right)+\mathcal{O}\left(m^{-6}\right) .
\end{aligned}
$$

(Before proceeding further, note that this ansatz captures the generic class of solutions where a diagonal $R_{\nu}^{\mu}(g)$ implies a diagonal $S_{\nu}^{\mu}$. One may also find special bimetric solutions where a diagonal $R_{\nu}^{\mu}(g)$ corresponds to a non-diagonal $S_{\nu}^{\mu}$. To see this, note that Equation (15) has the generic form $R_{\nu}^{\mu}=$ $\sum_{r} q_{r}\left(S^{r}\right)_{\nu}^{\mu}$, where the scalars $q_{r}$ are functions of the $\beta_{n}$ and of $e_{n}(S)$. It is possible to construct non-diagonal $S$, such that $\sum_{r} q_{r} S^{r}$ is diagonal. The off-diagonal structure depends on the choice of the $\beta_{n}$, and this is not possible in models with only one out of $\beta_{1}, \beta_{2}$ and $\beta_{3}$ nonvanishing [50]. For solutions of this type, see, for example, [70]. ) The coefficients $a, b_{i}$ and $c_{i}$ are determined by plugging this into Equation (15) and matching the coefficients of different powers of $P_{\nu}^{\mu}$. The details are given in the Appendix. The result can be presented simply in terms of the sums,

$$
s_{k} \equiv \sum_{n=k}^{d-1}\left(\begin{array}{c}
d-1-k \\
n-k
\end{array}\right) \beta_{n} a^{n}, \quad k=0,1,2,3 \ldots
$$

The coefficient $a$ is determined in terms of $\beta_{n}$ as a solution of the polynomial equation,

$$
s_{0} \equiv \sum_{n=0}^{d-1}\left(\begin{array}{c}
d-1 \\
n
\end{array}\right) \beta_{n} a^{n}=0 .
$$


Non-real solutions are not excluded as long as the parameters in the final action are real. For the $b_{i}$, one obtains,

$$
b_{2}=0, \quad b_{1}=\frac{a}{s_{1}} .
$$

where $s_{1}$ is defined in Equation (24). Finally, the $c_{i}$ are given by,

$$
c_{1}=-c_{2}=\frac{a s_{2}}{s_{1}^{3}}, \quad c_{3}=-c_{4}=-\frac{a s_{2}}{2(d-1) s_{1}^{3}} .
$$

These coefficients exist only if $s_{1} \neq 0$ (the case $s_{1}=0$ requires separate treatment and implies Ricci flat $g_{\mu \nu}, c f$. Equation (95)), and in terms of them, the solution for $S_{\nu}^{\mu}$ to $\mathcal{O}\left(m^{-4}\right)$ reads,

$$
S_{\nu}^{\mu}=a \delta_{\nu}^{\mu}+\frac{a}{s_{1} m^{2}} P_{\nu}^{\mu}+\frac{a s_{2}}{s_{1}^{3} m^{4}}\left[\left(P_{\nu}^{\mu 2}-P P_{\nu}^{\mu}\right)+\frac{1}{d-1} e_{2}(P) \delta_{\nu}^{\mu}\right]+\mathcal{O}\left(m^{-6}\right) .
$$

Note that the higher order curvature terms are suppressed by the mass scale,

$$
m^{2} s_{1}=m^{2} \sum_{n=1}^{d-1}\left(\begin{array}{l}
d-2 \\
n-1
\end{array}\right) \beta_{n} a^{n} .
$$

This is proportional to the Fierz-Pauli mass Equation (18) of the massive spin-2 fluctuation on proportional backgrounds $f_{\mu \nu}=a^{2} g_{\mu \nu}$ and with vanishing cosmological constant. (For backgrounds given by $f_{\mu \nu}=a^{2} g_{\mu \nu}$, the cosmological constant $\Lambda_{g}$ in the $g$-equation is given by $s_{0}$. In the present setup, $a$ must be chosen, such that $s_{0}=0$. For backgrounds $f_{\mu \nu}=c^{2} g_{\mu \nu}$, with $c \neq a$, the cosmological constant is no longer given by $s_{0}$.) Thus, the perturbative expansion becomes more accurate for large Fierz-Pauli mass, although the procedure does not really correspond to integrating out the massive mode of the bimetric theory. Rather, we are eliminating $f_{\mu \nu}$, which is not a mass eigenstate.

This procedure can be continued to compute the solution for $S_{\nu}^{\mu}$ to any order in $m^{-2}$. Here, we are mostly interested in the quadratic curvature terms in the higher-derivative action for $g_{\mu \nu}$, and hence, it suffices to determine the solution for $S_{\nu}^{\mu}$ to second order. As in the $\beta_{1}$ model, matter sources can be taken into account through the replacement Equation (22) in the final solution. It is easy to verify that for $\beta_{2}=\cdots=\beta_{d-1}=0$, one reproduces the exact result for the $\beta_{1}$ model obtained above as in this case, $s_{n}=0$ for $n \geq 2$.

\subsection{Higher Derivative Gravity from Bimetric Theory}

In this section, we eliminate $f_{\mu \nu}$ in the bimetric action using Equation (28) to obtain a higher curvature theory for $g_{\mu \nu}$. We explicitly retain only quadratic curvature terms and neglect the source terms, which can always be reinstated by the replacement Equation (22).

Let us begin by eliminating $S$ from the bimetric interaction potential. For this purpose, it is convenient to first express the potential in terms of,

$$
M_{\nu}^{\mu} \equiv \frac{1}{a} S_{\nu}^{\mu}-\delta_{\nu}^{\mu}
$$

Then, using the properties of the $e_{n}(S)$, one has,

$$
2 m^{2} \sqrt{g} \sum_{n=0}^{d} \beta_{n} e_{n}(S)=2 m^{2} \sqrt{g} \sum_{n=0}^{d} \alpha_{n} e_{n}(M)
$$


where we have defined,

$$
\alpha_{n}=\sum_{k=n}^{d}\left(\begin{array}{l}
d-n \\
k-n
\end{array}\right) a^{k} \beta_{k}
$$

Since $M$ directly starts at order $m^{-2}$, only $e_{n}(M)$ with $n \leq 2$ contribute to order $m^{-2}$ in the potential. Explicitly, $e_{0}(M)=1$ while $e_{1}(M)$ and $e_{2}(M)$ are given by,

$$
\begin{aligned}
& e_{1}(M)=\frac{1}{s_{1} m^{2}} e_{1}(P)-\frac{s_{2}}{s_{1}^{3} m^{4}} \frac{d-2}{d-1} e_{2}(P)+\mathcal{O}\left(m^{-6}\right), \\
& e_{2}(M)=\frac{1}{s_{1}^{2} m^{4}} e_{2}(P)+\mathcal{O}\left(m^{-6}\right) .
\end{aligned}
$$

Hence, the potential is given by,

$$
\begin{aligned}
& 2 m^{2} \sqrt{g} \sum_{n=0}^{d} \beta_{n} e_{n}(S)= \\
& \quad 2 m^{2} \sqrt{g}\left(\alpha_{0}+\frac{\alpha_{1}}{s_{1} m^{2}} e_{1}(P)+\frac{1}{s_{1}^{3} m^{4}}\left(s_{1} \alpha_{2}-s_{2} \alpha_{1} \frac{d-2}{d-1}\right) e_{2}(P)\right)+\mathcal{O}\left(m^{-4}\right) .
\end{aligned}
$$

Using Equation (21), it is straightforward to express $e_{n}(P)$ in terms of curvatures of $g_{\mu \nu}$,

$$
e_{1}(P)=\frac{d-2}{2(d-1)} R, \quad e_{2}(P)=\frac{1}{2}\left(\frac{d}{4(d-1)} R^{2}-R^{\mu \nu} R_{\mu \nu}\right) .
$$

Then, the bimetric potential expressed in terms of curvatures becomes,

$$
\begin{aligned}
2 m^{2} \sqrt{g} \sum_{n=0}^{d} \beta_{n} e_{n}(S)=2 m^{2} & \sqrt{g}\left[\alpha_{0}+\alpha_{1} \frac{d-2}{d-1} \frac{1}{2 s_{1} m^{2}} R\right. \\
& \left.+\frac{s_{1} \alpha_{2}-s_{2} \alpha_{1} \frac{d-2}{d-1}}{2 s_{1}^{3} m^{4}}\left(\frac{d}{4(d-1)} R^{2}-R^{\mu \nu} R_{\mu \nu}\right)\right]+\mathcal{O}\left(m^{-4}\right) .
\end{aligned}
$$

Let us now consider the kinetic term $\sqrt{f} R(f)$. In contrast with the bimetric potential, which produces polynomials only in curvatures of $g_{\mu \nu}$, the kinetic term also produces terms with covariant derivatives acting on curvatures. At quartic order in derivatives, the Bianchi identity can be used to show that the only possible such term is $\nabla^{2} R$. However, it will turn out in the following that the coefficient of this term is zero for all $\beta_{n}$. Terms involving derivatives of curvatures only start to appear at cubic order.

The solution for $f_{\mu \nu}$ is obtained from Equation (28) using $f_{\mu \nu}=g_{\mu \rho}\left(S^{2}\right)^{\rho}{ }_{\nu}$ and reads, to first order in curvatures,

$$
f_{\mu \nu}=a^{2} g_{\mu \nu}+\frac{2 a^{2}}{s_{1} m^{2}} P_{\mu \nu}+\mathcal{O}\left(m^{-4}\right) .
$$

Note that in $\sqrt{f} R(f)$, only terms up to this order contribute to the quadratic curvature terms. Expanded to first order in powers of $m^{-2}$, the inverse of $f_{\mu \nu}$ is then given by,

$$
\left(f^{-1}\right)^{\mu \nu}=a^{-2} g^{\mu \nu}-\frac{2}{a^{2} s_{1} m^{2}} P^{\mu \nu}+\mathcal{O}\left(m^{-4}\right) .
$$

Using the curvature relations (see, e.g., [71]),

$$
\begin{aligned}
R_{\mu \nu}(f) & =R_{\mu \nu}(g)+2 \nabla_{[\mu} C_{\alpha] \nu}{ }^{\alpha}-2 C_{\nu[\mu}{ }^{\beta} C_{\alpha] \beta}{ }^{\alpha}, \\
C_{\mu \nu}{ }^{\alpha} & =\frac{1}{2}\left(f^{-1}\right)^{\alpha \rho}\left(\nabla_{\mu} f_{\nu \rho}+\nabla_{\nu} f_{\mu \rho}-\nabla_{\rho} f_{\mu \nu}\right),
\end{aligned}
$$


we can express the curvatures of $f_{\mu \nu}$ in terms of curvatures and connections of $g_{\mu \nu}$. For the $f_{\mu \nu}$ obtained above, this gives,

$$
R_{\mu \nu}(f)=R_{\mu \nu}(g)+\frac{2}{s_{1} m^{2}}\left(\nabla_{\mu} \nabla_{\nu} P_{\alpha}^{\alpha}-\nabla^{\rho} \nabla_{\nu} P_{\rho \mu}-\nabla^{\rho} \nabla_{\mu} P_{\rho \nu}+\nabla^{2} P_{\mu \nu}\right)+\mathcal{O}\left(m^{-4}\right) .
$$

The $C_{\mu \nu}{ }^{\alpha}$ contribute the terms linear in $P_{\mu \nu}$, and in $R(f)=f^{\mu \nu} R_{\mu \nu}(f)$, these drop out due to the usual Bianchi identity,

$$
\begin{aligned}
R(f) & =a^{-2} R(g)-\frac{2}{a^{2} s_{1} m^{2}} P^{\mu \nu} R_{\mu \nu}(g)+\frac{4}{a^{2} s_{1} m^{2}}\left(\nabla^{2} P^{\alpha}{ }_{\alpha}-\nabla^{\mu} \nabla^{\nu} P_{\mu \nu}\right)+\mathcal{O}\left(m^{-4}\right) \\
& =a^{-2} R(g)-\frac{2}{a^{2} s_{1} m^{2}}\left(R^{\mu \nu} R_{\mu \nu}-\frac{1}{2 d-2} R^{2}\right)+\mathcal{O}\left(m^{-4}\right),
\end{aligned}
$$

where we have used the identity $\nabla^{2} P^{\alpha}{ }_{\alpha}-\nabla^{\mu} \nabla^{\nu} P_{\mu \nu}=0$, which follows from the Bianchi identity. As promised, the terms involving derivatives acting on curvatures have dropped out. Furthermore, using $\operatorname{det}(1+A)=\sum_{n} e_{n}(A)$ and retaining the first two terms, we have,

$$
\sqrt{f}=a^{d} \sqrt{g} \sum_{n=0}^{d} e_{n}\left(\frac{P}{s_{1} m^{2}}\right)=a^{d} \sqrt{g}\left(1+\frac{1}{2 s_{1} m^{2}} \frac{d-2}{d-1} R(g)\right)+\mathcal{O}\left(m^{-4}\right) .
$$

Then, the kinetic terms become,

$$
\begin{aligned}
\sqrt{g} R(g) & +\alpha^{d-2} \sqrt{f} R(f) \\
= & \sqrt{g}\left[\left(1+(\alpha a)^{d-2}\right) R(g)-\frac{2(\alpha a)^{d-2}}{s_{1} m^{2}}\left(R^{\mu \nu} R_{\mu \nu}-\frac{d}{4(d-1)} R^{2}\right)\right]+\mathcal{O}\left(m^{-4}\right) .
\end{aligned}
$$

Note that this has the same structure as the potential terms. Finally, combining Equations (36) and (43), we obtain the higher derivative Lagrangian for $g_{\mu \nu}$ to quadratic order in curvatures,

$$
\mathcal{L}_{(2)}^{\mathrm{HD}}(g)=m_{g}^{d-2} \sqrt{g}\left[\Lambda+c_{R} R(g)-\frac{c_{R R}}{m^{2}}\left(R^{\mu \nu} R_{\mu \nu}-\frac{d}{4(d-1)} R^{2}\right)\right]+\mathcal{O}\left(m^{-4}\right) .
$$

This coincides with Equation (1), and the parameters are given by,

$$
\begin{aligned}
\Lambda & =-2 m^{2} \alpha_{0}, \\
c_{R} & =1+(\alpha a)^{d-2}-\frac{\alpha_{1}}{s_{1}} \frac{d-2}{d-1}, \\
c_{R R} & =\frac{1}{s_{1}^{2}}\left(2(\alpha a)^{d-2} s_{1}-\alpha_{2}+\frac{s_{2}}{s_{1}} \frac{d-2}{d-1} \alpha_{1}\right) .
\end{aligned}
$$

The theory can also be written in a more compact form,

$$
\mathcal{L}_{(2)}^{\mathrm{HD}}(g)=m_{g}^{d-2} \sqrt{g}\left[\Lambda+c_{R}^{\prime} e_{1}(P)+c_{R R}^{\prime} e_{2}(P)\right]+\mathcal{O}\left(m^{-4}\right)
$$

where $c_{R}^{\prime}=2(d-1) c_{R} / d-2$ and $c_{R R}^{\prime}=2 c_{R R} / m^{2}$.

An identity that follows from Equations (24) and (32) is,

$$
s_{n}=\alpha_{n}-\alpha_{n+1} .
$$


Since $s_{0}=0$, this implies $\alpha_{0}=\alpha_{1}$ and also gives an expression for the coefficients in terms of the $\alpha_{n}$ or using Equation (32) in terms of the $\beta_{n}$. Note that $\mathcal{L}_{(2)}^{\mathrm{HD}}$ depends on only three combinations of the $d+1$ parameters $\beta_{n}$. To see all of the free parameters of the bimetric theory, one needs to consider higher order curvature terms in $\mathcal{L}^{\mathrm{HD}}$. The truncation is accurate only in the low curvature regime $R(g)<<m^{2}$. It is worth pointing out, however, that although the HD Lagrangian is in general an infinite expansion in curvatures, the number of parameters determining this expansion is just the $d+1$ parameters $\beta_{n}$ together with $\alpha=m_{f} / m_{g}$.

\section{The Ghost Issue and Relevance to New Massive Gravity}

The higher derivative gravity Lagrangian (44) propagates seven modes in $d=4$, comprising two modes of a healthy massless graviton and five modes of a massive spin-2 field, which is a ghost. This is in contrast to the original bimetric theory in which all seven modes are healthy. The masses of the spin-2 fields are also not equal in the two theories. Of course, in the HD gravity case, one is working with a truncated action where the mass of the problematic excitation is related to the truncation scale. Therefore, it is expected that the ghost problem is an artifact of the truncation.

To illustrate this, in the Appendix, we consider two examples, the linearized version of bimetric theory and a very similar, but much simpler theory of two scalars fields. In the absence of interactions, these theories can be manipulated explicitly. In both the spin- 0 and spin- 2 examples, one of the fields can be eliminated to explicitly obtain a closed form higher derivative action for the remaining field, including appropriate source couplings inherited from the original bi-field theory. The action contains up to six-derivative terms. To check for ghosts, we compute the vacuum persistence amplitude in the higher derivative theory. This turns out to be exactly the same as the one computed in the associated bi-field theory. The equivalence implies that the untruncated HD theory is ghost free and has the right mass poles. However, if this theory is now truncated to four-derivative terms, the massive field becomes a ghost, and its mass shifts away from the correct value, thus illustrating the point.

In the presence of bimetric interactions, the corresponding HD gravity obtained in a perturbative manner will not have a finite number of terms, and it is not feasible to write such a theory in a closed form. Considering this, $S_{(2)}^{\mathrm{HD}}$, Equation (1), is usable only as an effective theory at energy scales below the mass pole, and the $R^{2}$ terms must be treated only perturbatively. The present analysis, however, implies that $S_{(2)}^{\mathrm{HD}}$ has a minimal ghost-free completion in the form of an HR bimetric theory. Even simpler, since at the four-derivative level, the HD action has only three independent parameters, its structure can be reproduced by the $\beta_{1}$ model alone. This will also avoid the issue raised in the comment below Equation (23). The equations of motion derived from the full $S^{\mathrm{HD}}$ differ from the corresponding bimetric equation (obtained on eliminating $f_{\mu \nu}$ between Equations (15) and (16)) by the extra operator $\delta f / \delta g$, as discussed earlier. The truncation procedure mixes this operator with the HD equation of motion, and the similarity with the bimetric equation is no longer obvious.

The correspondence between the HR bimetric theory and the action $S^{\mathrm{HD}}$ may also be regarded as a reconfirmation that the bimetric theory propagates only seven modes and no Boulware-Deser ghost [64].

One can also consider the implications of this correspondence to new massive gravity (NMG). There has been some work to discover and understand ghost-free higher dimensional generalizations 
of the NMG models, with no success. NMG corresponds to $\mathcal{L}_{(2)}^{\mathrm{HD}}$ for $d=3$ and $m_{g} c_{R}<0$, in which case the theory contains a healthy massive spin-2 mode. With $m_{g} c_{R}<0$, the massless spin-2 mode becomes a ghost, but in $d=3$, this mode does not propagate. This way, one ends up with a healthy theory of a massive spin-2 field. However, this mechanism of removing the ghost is peculiar to $d=3$, and attempts to generalize NMG beyond $d=3$ have so far remained largely unsuccessful [9]. The present analysis indicates that the bimetric theory is indeed one (possibly the only) minimal completion of NMG, providing a ghost-free generalization to all dimensions.

\section{Conformal Gravity from Partially Massless Bimetric Theory}

Recently, the problem of finding nonlinear realizations of partial masslessness, first observed in the linear Fierz-Pauli theory in dS backgrounds [57], has attracted some attention [3,4,59,62,63,68]. One such nonlinear candidate is the PM bimetric theory that exists only in three and four dimensions and that directly contains the original linear PM theory in dS backgrounds [3,4]. In these works, it was not established that away from dS backgrounds, this theory still contains six modes (in $d=4$ ), rather than the generic seven modes of the HR bimetric theory. Another possible PM candidate is conformal gravity, whose spectrum around dS backgrounds was observed to be similar to the linear PM spectrum [21]. CG in general propagates six modes, but the spectrum is plagued by the usual ghosts of HD gravity. On the face of it, these are very different approaches to the PM issue.

It is now easy to check that the HD gravity corresponding to the PM bimetric theory is indeed related to conformal gravity. What is more, it turns out that at the four-derivative level, the PM bimetric theory and CG have exactly the same equations of motion (which is not the case for the non-PM actions considered above). Hence, the PM bimetric theory is indeed a ghost-free extension of conformal gravity. Conversely, this provides further evidence that the PM bimetric theory propagates six modes even away from dS backgrounds.

\subsection{The Correspondence in $d=4$}

The partially massless bimetric theory in $d=4$ is specified by setting $[3,4]$,

$$
\beta_{1}=\beta_{3}=0, \quad \alpha^{4} \beta_{0}=3 \alpha^{2} \beta_{2}=\beta_{4} .
$$

Then, Equations (24), (25) and (32) give,

$$
a^{2}=-\frac{1}{\alpha^{2}}, \quad s_{1}=-\frac{2 \beta_{2}}{\alpha^{2}}, \quad \alpha_{0}=\alpha_{1}=0, \quad \alpha_{2}=\frac{2 \beta_{2}}{\alpha^{2}} .
$$

and the solution for $f_{\mu \nu}$ takes the form,

$$
f_{\mu \nu}=-\frac{1}{\alpha^{2}} g_{\mu \nu}+\frac{1}{\beta_{2} m^{2}} P_{\mu \nu}+\mathcal{O}\left(m^{-4}\right) .
$$

Using these parameter values in Equation (45), the higher derivative Lagrangian Equation (44) reduces to,

$$
\mathcal{L}^{\mathrm{CG}}=-\frac{\alpha^{2} m_{g}^{2}}{2 \beta_{2} m^{2}} \sqrt{g}\left(R^{\mu \nu} R_{\mu \nu}-\frac{1}{3} R^{2}\right)+\mathcal{O}\left(m^{-4}\right)
$$


Restricted to quadratic order in curvatures, this is the well-known action for conformal gravity. It is invariant under Weyl scalings of the metric, and as a result, it contains only four propagating massive modes, in addition to two massless modes.

The relation between conformal gravity and PM bimetric theory is constrained enough that the above reasoning can be carried out in reverse: demanding that the higher derivative action Equation (44) reduces to conformal gravity at the quadratic level (that is, $\alpha_{0}=0, c_{1}=0$ ) fixes the parameters of the bimetric theory to their PM values. Explicitly, $\alpha_{0}=0$ and $s_{0}=0$ Equation (25) imply $\alpha_{1}=0$, since $\alpha_{0}-\alpha_{1}=s_{0}$, Equation (47). Hence, in order for the Einstein-Hilbert term to vanish, that is $c_{1}=0$, we must have $a^{2}=-\alpha^{-2}$. Then, the $s_{0}=0$ equation, Equation (25), becomes,

$$
\beta_{0}+\frac{3 i}{\alpha} \beta_{1}-\frac{3}{\alpha^{2}} \beta_{2}-\frac{i}{\alpha^{3}} \beta_{3}=0 .
$$

Furthermore, the condition $\alpha_{0}=0$ reads, Equation (32),

$$
\beta_{0}+\frac{4 i}{\alpha} \beta_{1}-\frac{6}{\alpha^{2}} \beta_{2}-\frac{4 i}{\alpha^{3}} \beta_{3}+\frac{1}{\alpha^{4}} \beta_{4}=0 .
$$

The real and imaginary parts must vanish simultaneously, which gives Equation (48) as the unique solution. This establishes the correspondence between CG and PM bimetric theory.

\subsection{A Step Further: Equivalence between CG and PM Bimetric Theory}

As we have seen, in general, the bimetric equations of motion and the corresponding HD gravity equations differ by an operator $\delta f / \delta g$. On truncating to four-derivative terms, as in $S_{(2)}^{\mathrm{HD}}$, one finds that the truncated theories are not equivalent. An exception is the PM case, as shown below.

The equations of motion arising from the conformal gravity action Equation (3) correspond to setting the Bach tensor $B_{\mu \nu}$ of $g_{\mu \nu}$ to zero [16],

$$
B_{\mu \nu} \equiv-\nabla^{2} P_{\mu \nu}+\nabla^{\rho} \nabla_{(\mu} P_{\nu) \rho}+W_{\rho \mu \nu \sigma} P^{\rho \sigma}=0 .
$$

In this expression, the four-dimensional Weyl tensor $W_{\rho \mu \nu \sigma}$ is given by:

$$
W_{\rho \mu \nu \sigma} \equiv R_{\rho \mu \nu \sigma}+g_{\mu[\nu} R_{\sigma] \rho}-g_{\rho[\nu} R_{\sigma] \mu}+\frac{1}{3} g_{\rho[\nu} g_{\sigma] \mu} R
$$

Let us now consider the corresponding equation in the PM bimetric theory. The bimetric $g$-equation has already been solved to determine $S$ Equation (28) and $f_{\mu \nu}$ Equation (37). We substitute these in the bimetric $f$-equation, Equation (16), using the expression Equations (40) and (41) for the curvatures of $f_{\mu \nu}$. This gives a higher derivative equation for $g_{\mu \nu}$. On restricting to the PM parameter values, Equation (48), it is straightforward to show that the cosmological constant term and the Einstein tensor drop out, and the equation reduces to,

$$
-B_{\mu \nu}+\mathcal{O}\left(m^{-4}\right)=0
$$

Hence, the PM bimetric equation, evaluated to lowest non-trivial order in $R_{\mu \nu}(g) / m^{2}$, coincides with the $\mathrm{CG}$ equation (this is, of course, consistent with the general analysis of the previous sections and 
follows from the fact that, in the PM case, only the lowest order term in $\delta f / \delta g \sim a^{2}=-1 / \alpha^{2}$ contributes to the relations between the equations of motion).

In other words, the conformal gravity Equation (54) is the genuine low curvature limit of the PM bimetric theory. The difference is that while some of the propagating modes arising from this equation have negative norms in the CG framework, they appear as healthy non-ghost modes in the HR bimetric setup. This implies that the PM bimetric theory can be regarded as a ghost-free extension of conformal gravity.

This equivalence also has implications for the PM bimetric theory that has been identified as the only possible nonlinear generalization of the linear PM theory in the HR bimetric setup [3]. It is known that this theory has six propagating modes around dS backgrounds, a massless spin-2 field and the four-component PM field. The seventh mode, which exists in a generic HR bimetric theory, is eliminated by a new gauge invariance [57]. The question is if away from dS backgrounds, this theory will still have a gauge invariance and, consequently, propagate only six modes. Some indirect evidence for this has been discussed in $[3,4]$. The relation to conformal gravity found here provides direct evidence for the proposal, as it establishes that, in the small curvature limit, the PM bimetric theory indeed propagates only six modes around any background.

The Bach Equation (54) propagates six modes not only around dS [21], but also around a flat background [20]. Thus, an obvious question is whether the higher derivative corrections to the Bach equation in Equation (56) can alter the propagator for the linear fluctuation around a flat background. We will argue now that this is not the case, and the propagator for the fluctuation $\delta g_{\mu \nu}$ around flat space is not affected by the higher derivative corrections to Equation (56). Namely, consider the solution for $f_{\mu \nu}$ in terms of $g_{\mu \nu}$ given in Equation (50). Taking into account the fact that the terms nonlinear in $P_{\mu \nu}(g)$ do not contribute to the linearized equation because $P_{\mu \nu}(\eta)=0$, only two derivatives are acting on $\delta g_{\mu \nu}$. It is then also clear that when this solution is plugged into the $f_{\mu \nu}$ equation of motion, the resulting linearized equation will contain at most two more derivatives acting on $\delta g_{\mu \nu}$ coming from the Einstein tensor for $f_{\mu \nu}$. Hence, the linearized equation for $g_{\mu \nu}$ around flat space is fourth order in derivatives. The fluctuation equation obtained in this way is of course the same as the linearized version of Equation (56), in which all four-derivative terms come from the Bach tensor. Hence, the propagator around flat space is obtained from the Bach tensor alone and is therefore identical to the propagator in conformal gravity. A similar argument is valid for dS backgrounds, confirming the result obtained in the bimetric formulation that a linear gauge symmetry is present around these backgrounds.

Furthermore, in the small curvature limit, the symmetry transformations of the bimetric fields $g_{\mu \nu}$ and $f_{\mu \nu}$ follow from the Weyl invariance of conformal gravity. Thus, to linear order in curvatures and in the gauge parameter, and modulo coordinate transformations, we get,

$$
g_{\mu \nu}^{\prime}=g_{\mu \nu}+\phi g_{\mu \nu}, \quad f_{\mu \nu}^{\prime}=f_{\mu \nu}-\frac{\phi}{\alpha^{2}} g_{\mu \nu}-\frac{1}{\beta_{2} m^{2}} \nabla_{\mu}^{g} \nabla_{\nu}^{g} \phi
$$

Let us now consider the relation between dS backgrounds in conformal gravity and in the PM bimetric theory. The CG equation Equation (54) admits dS solutions for which $R_{\mu \nu}(\bar{g})=\bar{g}_{\mu \nu} \Lambda$ (or $P_{\mu \nu}(\bar{g})=$ $\frac{1}{3} \bar{g}_{\mu \nu} \Lambda$ ). The cosmological constant is not determined by the theory and can be changed to any value by constant Weyl scalings of the metric, which are symmetries of the theory. 
This solution of CG is mapped to bimetric theory by Equation (50). It turns out that on dS backgrounds, all higher curvature corrections to Equation (50) vanish, and the terms explicitly written out give,

$$
\bar{f}_{\mu \nu}=\left(-\frac{1}{\alpha^{2}}+\frac{\Lambda}{3 \beta_{2} m^{2}}\right) \bar{g}_{\mu \nu} .
$$

Writing this as $\bar{f}_{\mu \nu}=c^{2} \bar{g}_{\mu \nu}$, we obtain,

$$
\Lambda=m^{2}\left(\frac{1+c^{2} \alpha^{2}}{\alpha^{2}}\right) 3 \beta_{2},
$$

which is indeed the expression for the cosmological constant in terms of parameters of the PM bimetric theory. In this setup, $c$ is an arbitrary gauge-dependent parameter. The flat space limit corresponds to $c^{2}=-1 / \alpha^{2}$. In the bimetric case, it is possible to express the theory in terms of variables, such that all observables become independent of $c$ [4].

Let us consider conformal gravity solutions away from dS spacetimes, that is, when $R_{\mu \nu}(g)$ is not proportional to $g_{\mu \nu}$. Such solutions, which are Bach flat, but not conformally Einstein, are known to exist, see, e.g., [72,73]. Then, from Equation (50), it follows that in the bimetric setup, the field $f_{\mu \nu}$ is not proportional to $g_{\mu \nu}$. In such cases, the bimetric spectrum does not generically decompose into a massless and a massive spin-2 field [29]. Consequently, on the conformal gravity side, too, the spectrum is not expected to follow such a decomposition away from dS backgrounds.

\section{Discussions}

Our results are summarized in the Introduction section. Here, we would like to first reemphasize two issues.

(1) The analysis in this paper shows that the HR bimetric theory captures the essential features of higher derivative gravity action Equation (1), while at the same time avoiding the spin-2 ghost problem. The correspondence between the two theories found here is not a complete equivalence of equations of motion, but can still be used to generate higher derivative completions of the four-derivative gravity actions.

(2) The equation of motion in the candidate PM bimetric theory at the four-derivative level was shown to coincide with the Bach equation of conformal gravity. While this result was motivated by the general correspondence between bimetric and HD gravity actions, it turns out to bypass the general correspondence and, in fact, was an equivalence at the level of equations of motion. As a result, it has genuine consequences for the bimetric PM proposal, as discussed in the paper.

We also reiterate that the analysis of [52,54], on acausal propagation and the absence of a nonlinear PM symmetry in massive gravity, relies on the presence of a non-dynamical metric and, as it stands, breaks down for the HR bimetric theory. For this reason, we have emphasized the distinctions between the HR bimetric models and the dRGT massive gravity models.

One may also consider alternative formulations and generalizations of the PM bimetric model in terms of vielbeins. Introducing vielbeins $e^{a}{ }_{\mu}$ and $\tilde{e}_{\mu}^{a}$ for $g_{\mu \nu}$ and $f_{\mu \nu}$, respectively, the square-root matrix 
can be expressed as $\left(\sqrt{g^{-1} f}\right)_{\nu}^{\mu}=e_{c}^{\mu} \tilde{e}^{c}{ }_{\nu}$, provided the vielbeins satisfy the symmetrization condition, $\eta_{a c} \tilde{e}_{\rho}^{c} e_{b}^{\rho}=e_{a}^{\rho} \tilde{e}_{\rho}^{c} \eta_{c b}$, or in matrix form,

$$
\eta \tilde{e} e^{-1}=\left(\eta \tilde{e} e^{-1}\right)^{T}
$$

In general, this condition can be implemented through a Lorentz transformation, though there are caveats $[74,75]$ to this. However, it has also been shown that the bimetric theory expressed in terms of unconstrained vielbeins contains the above symmetrization condition as part of its equations of motion [76], in the sense that this condition is always a solution. This work also formulates more general multivielbein theories that, with their equivalent metric formulations [77], open the way for investigating PM theories with more than one extra gauge invariance.

It would also be interesting to consider the conformal gravity analogues of the PM bimetric theory in higher dimensions. It is known that on adding Lanczos-Lovelock terms to the bimetric action, one can obtain candidate PM bimetric theories in higher dimensions, in particular for $d=6$ and $d=8$ [4]. One then expects that on eliminating $f_{\mu \nu}$, the corresponding equations of motion, to lowest order in the curvature expansion, coincide with the equations of the recently constructed conformal gravity theories in six [78,79] and eight dimensions [80].

Note: Since this paper first appeared as a preprint, some works have appeared claiming negative results for the possibility of a nonlinear extension of the PM theory in various setups [81-83]. However, these results do not yet extend to the present bimetric setup. In particular, [81] claims that the relation between the PM bimetric candidate and an extension of conformal gravity, as found in this paper, is obtained only at the level of the action (as in Section 4.1); hence, it cannot be treated as an equivalence between the corresponding equations of motion. This claim is astonishingly incorrect. The authors in [81] have chosen to ignore that this issue had already been explicitly raised and addressed in the present paper, where, in Section 4.2, the relation is established at the level of bimetric equations of motion. This issue was then re-emphasized when summarizing the results in Section 1.2 and, again, when discussing them in Section 5.

On the other hand, [82] carries out an interesting analysis starting with cubic interactions of a PM field, but as argued in the Appendix of that paper, these cubic terms indeed vanish for the PM bimetric candidate. Therefore, it is not obvious that, in spite of this, the analysis of [82] would still apply to bimetric theory. Arguments that rule out PM symmetry in bimetric theory, based on results from massive gravity, have been dealt with in [50]. In a forthcoming work, we will show that the transformation Equation (57) can be extended to at least six-derivative order [84].

\section{Acknowledgments}

We would like to thank Shailesh Lal and Bo Sundborg for discussions.

\section{Author Contributions}

All of the authors have contributed an equal amount to all of the underlying analytical work as well as the final completion of this work into article form. All authors have read and approved the final manuscript. 


\section{Conflicts of Interest}

The authors declare no conflict of interest.

\section{A. Higher Derivative Treatment of Free Massive Spin-0 and Spin-2 Fields}

To better understand the relation between bimetric theory and higher derivative gravity and to resolve the ghost problem of the latter, we first illustrate the procedure in a much simpler scalar field analogue of the bimetric action. Then, we consider the linearized bimetric theory to emphasize the parallel with the scalar field case.

\section{A.1. Higher Derivative Treatment of Massive Scalars}

Consider the theory of two scalar fields $\phi$ and $\psi$ with a $\phi \psi$ mixing,

$$
S=\int d^{d} x\left[-\frac{1}{2} \partial_{\mu} \phi \partial^{\mu} \phi-\frac{1}{2} \partial_{\mu} \psi \partial^{\mu} \psi-\frac{\mu^{2}}{2}(\phi+\psi)^{2}-V(\phi, \psi)-\phi J_{\phi}-\psi J_{\psi}\right] .
$$

It describes a pair of massless and massive scalars, $\Phi_{0}=\phi-\psi, \Phi_{m}=\phi+\psi$, in a non-diagonal basis. Conventionally, this theory is analyzed in terms of the mass eigenstates. Here, we want to discuss two approaches to recast Equation (61) as a higher derivative theory of a single field, with emphasis on the ghost problem. However, for later reference, we first review the usual discussion of ghosts in terms of the vacuum persistence amplitude. For $V=0$, the field equations are,

$$
\begin{aligned}
& \frac{\delta S}{\delta \phi}=0 \quad \Rightarrow \quad \square \phi-\mu^{2}(\phi+\psi)=J_{\phi}, \\
& \frac{\delta S}{\delta \psi}=0 \quad \Rightarrow \quad \square \psi-\mu^{2}(\phi+\psi)=J_{\psi},
\end{aligned}
$$

On adding and subtracting, they diagonalize to,

$$
\square \Phi_{0}=J_{0}, \quad\left(\square-2 \mu^{2}\right) \Phi_{m}=J_{m},
$$

with:

$$
\Phi_{0}=\phi-\psi, \quad \Phi_{m}=\phi+\psi, \quad J_{0}=J_{\phi}-J_{\psi}, \quad J_{m}=J_{\phi}+J_{\psi} .
$$

Hence, Equation (61) describes a massless scalar $\Phi_{0}$ and a massive one $\Phi_{m}$ of mass $2 \mu^{2}$. In particular, the solution for $\phi=\frac{1}{2}\left(\Phi_{0}+\Phi_{m}\right)$ is (we use the standard notation $\left(\square-m^{2}\right)^{-1} J(x)=\int d^{d} y G(x-y) J(y)$ with the Green's function $\left.G(x-y)=\left(\square-m^{2}\right)^{-1} \delta(x-y)=-\int d^{d} k \frac{e^{i k(x-y)}}{k^{2}+m^{2}+i \epsilon}\right)$,

$$
\phi=\frac{1}{2}\left[\square^{-1} J_{0}+\left(\square-2 \mu^{2}\right)^{-1} J_{m}+\Phi_{0}^{\mathrm{hom}}+\Phi_{m}^{\mathrm{hom}}\right],
$$

where $\Phi_{0, m}^{\text {hom }}$ are the homogeneous solutions of Equation (64). From Equation (61), on completing squares, one reads off the vacuum persistence amplitude,

$$
A=\int \mathcal{D} \phi e^{i S}=\exp \left(-\frac{i}{4} \int d^{d} x\left[J_{0}(x) \square^{-1} J_{0}(x)+J_{m}(x)\left(\square-2 \mu^{2}\right)^{-1} J_{m}(x)\right]\right)<1 .
$$


The propagators $i \square^{-1}$ and $i\left(\square-2 \mu^{2}\right)^{-1}$ have positive residues at the corresponding mass poles, which results in $A<1$, meaning that the theory is ghost free. In other words, the vacuum has decayed only into positive norm states due to interaction with the sources. In a theory that contains ghosts, it becomes possible to get $A>1$, implying a breakdown of unitarity and signaling vacuum decay into negative norm states. In the following, we will use this criterion to check for ghosts in free higher derivative theories where $A$ can be computed.

The potential $V(\phi, \psi)$ provides the interactions between the two mass eigenstates. At low energies, the massive mode $\Phi_{m}$ can be integrated out. In particular, for $V=0$, this gives a Lagrangian for the free massless field $\Phi_{0}$ with no knowledge of the massive mode. The analysis below does not involve integrating out fields in this way. Let us now consider the two approaches to obtaining higher derivative equations from Equation (61).

\section{A.2. First Approach: The Equivalent Higher Derivative Equations}

Instead of diagonalizing the equations of motion Equations (62) and (63), it is instructive to rewrite them as an equivalent higher derivative equation. The purpose is to illustrate that, given a higher derivative equation, the appearance of ghosts is not a forgone conclusion, but depends on the structure of the source terms and on the choice of action.

Let us eliminate $\psi$ between the equations of motion. The $\phi$-equation, Equation (62), is algebraic in $\psi$ and solves to,

$$
\psi=-\phi+\frac{1}{\mu^{2}}\left(\square \phi-J_{\phi}\right) .
$$

Such algebraic solutions can be obtained even for a non-zero $V(\phi, \psi)$, at least perturbatively in $1 / \mu^{2}$. Inserting this in Equation (63) gives a higher derivative equation (the same result follows on first solving Equation (63) for $\psi$ and substituting the outcome in Equation (62)),

$$
\square\left(\square-2 \mu^{2}\right) \phi=\frac{1}{2}\left[\left(\square-2 \mu^{2}\right) J_{0}+\square J_{m}\right] .
$$

The specific source structure in Equation (69) and the choice of action leading to it are crucial for the absence of a ghost. Had we ignored the sources in the above derivation and instead introduced a generic source coupling at the end, we would obtain $\square\left(\square-2 \mu^{2}\right) \phi=j$, corresponding to an action $\int d^{d} x\left[\frac{1}{2} \phi \square\left(\square-2 \mu^{2}\right) \phi-j \phi\right]$. This gives the correct mass poles, but implies that the massive mode is a ghost (in the naive propagator for this theory, $\frac{1}{k^{2}\left(k^{2}+m^{2}\right)}=\frac{1}{m^{2}}\left(\frac{1}{k^{2}}-\frac{1}{k^{2}+m^{2}}\right)$, the residues of the massless and massive poles have different signs, so in the analogue of Equation (67), $A$ is not always bounded by unity). Of course, it is not obvious why two propagating modes of different masses should couple to the same source with the same strength.

However, with the source structure of Equation (69), one gets the correct solution Equation (66). To check for the presence of ghosts, one needs to specify an action. The obvious procedure is to integrate out $\psi$ in Equation (61) (by completing the squares for $\psi$ or, equivalently, eliminating $\psi$ through the solution of its own equation of motion, and not Equation (68)). Then, for $V=0$, one obtains the bi-local higher derivative action for $\phi$, 


$$
\begin{gathered}
S_{b l}=\frac{1}{2} \int d^{d} x d^{d} y\left[\phi(x) G(x-y)\left[\square_{y}\left(\square_{y}-2 \mu^{2}\right) \phi(y)-\left(\square_{y}-2 \mu^{2}\right) J_{o}(y)+\square_{y} J_{m}(y)\right]\right. \\
\left.-\frac{1}{4}\left[J_{m}(x)-J_{0}(x)\right] G(x-y)\left[J_{m}(y)-J_{0}(y)\right]\right] .
\end{gathered}
$$

By construction, this has Equation (69) as the equation of motion and gives the vacuum persistence amplitude Equation (67), consistent with the absence of ghosts. Although the $J-J$ term in the second line does not contribute to the equation of motion, it is needed to get the correct ghost-free amplitude. Its pretense simply indicates that the interactions of the $\psi$ field cannot all be encoded in the structure of the $\phi$ action alone. To summarize, the above illustrates that a higher-derivative equation, like $\square\left(\square-2 \mu^{2}\right) \phi=0$, which normally implies a ghost, could actually be ghost free with the appropriate action and source couplings.

\section{A.3. Second Approach: A More General Higher Derivative Action}

Now, instead of using the algebraic solution $\psi\left(\phi, J_{\phi}\right)$ to eliminate $\psi$ from the equations of motion (first approach), we use it in the action to get $S\left[\phi, \psi\left(\phi, J_{\phi}\right)\right]$. This gives a six-derivative equation of motion for $\phi$, which is more general and contains the solutions of Equation (69) as a subsector. The analogue of this treatment, when applied to bimetric theory, reproduces the higher derivative gravity theories. The discussion here is intended to shed light on the emergence of the spin-2 ghost in higher derivative gravity.

Using the solution Equation (68) to eliminate $\psi$ in the action Equation (61) gives a six-derivative action,

$$
\begin{gathered}
S\left[\phi, \psi\left(\phi, J_{\phi}\right)\right]=\frac{1}{2 \mu^{4}} \int \mathrm{d}^{d} x\left[\phi \square\left(\square-\mu^{2}\right)\left(\square-2 \mu^{2}\right) \phi-2 \phi\left(\square-\mu^{2}\right)\left(\left(\square-\mu^{2}\right) J_{\phi}+\mu^{2} J_{\psi}\right)\right. \\
\left.+J_{\phi}\left(\square-\mu^{2}\right) J_{\phi}+2 \mu^{2} J_{\phi} J_{\psi}\right]
\end{gathered}
$$

The corresponding equation of motion is,

$$
\left.\frac{\delta S}{\delta \phi(x)}\right|_{\psi}+\left.\int d^{d} y \frac{\delta \psi(y)}{\delta \phi(x)} \frac{\delta S}{\delta \psi(y)}\right|_{\phi}=0
$$

The first term vanishes by virtue of Equation (68). Hence, one gets, $\left.\int d^{d} y \frac{\delta \psi(y)}{\delta \phi(x)} \frac{\delta S}{\delta \psi(y)}\right|_{\phi}=0$, which differs from $\frac{\delta S}{\delta \psi}=0$ by the extra operator, $\frac{\delta \psi(y)}{\delta \phi(x)}=\frac{1}{\mu^{2}}\left(\square_{y}-\mu^{2}\right) \delta(x-y)$, as follows form Equation (68). This gives the higher derivative equation,

$$
\left(\square-\mu^{2}\right)\left[\square\left(\square-2 \mu^{2}\right) \phi-\frac{1}{2}\left(\left(\square-2 \mu^{2}\right) J_{0}+\square J_{m}\right)\right]=0 .
$$

The terms within square brackets are the same as in Equation (69). Had we ignored the sources from the beginning and then inserted a generic source coupling at the end, we would obtain $\square\left(\square-\mu^{2}\right)\left(\square-2 \mu^{2}\right) \phi=j$, corresponding to an action $\int d^{4} x \frac{1}{2} \phi \square\left(\square-\mu^{2}\right)\left(\square-2 \mu^{2}\right) \phi-j \phi$. This would 
give a theory with three mass poles and a ghost mode. However, for the correct source structure, Equation (73) is equivalent to,

$$
\square\left(\square-2 \mu^{2}\right) \phi-\frac{1}{2}\left(\left(\square-2 \mu^{2}\right) J_{0}+\square J_{m}\right)=\chi^{\text {hom }},
$$

where $\chi^{\text {hom }}$ solves $\left(\square-\mu^{2}\right) \chi^{\text {hom }}=0$ and needs to be specified through two boundary conditions. The boundary conditions that give $\chi^{\text {hom }}=0$ specify the subsector of Equation (71) that is equivalent to Equation (61).

In general, the complete solution becomes,

$$
\phi=\frac{1}{2}\left[\square^{-1} J_{0}+\left(\square-2 \mu^{2}\right)^{-1} J_{m}\right]+\square^{-1}\left(\square-2 \mu^{2}\right)^{-1} \chi^{\mathrm{hom}}+\frac{1}{2}\left[\Phi_{0}^{\mathrm{hom}}+\Phi_{m}^{\mathrm{hom}}\right] .
$$

A non-vanishing $\chi^{\text {hom }}$ appears as an arbitrariness in the sources $J_{0}$ and $J_{m}$, but even then, only the two original mass poles of Equation (61) contribute to the solution.

To address the ghost issue, one can again compute the vacuum persistence amplitude $\int \mathcal{D} \phi e^{i S}$ by completing the squares for $\phi$ in the action Equation (71). The answer turns out to be exactly the same as Equation (67). In particular, there is no contribution from $\chi^{\text {hom }}$. Hence, the complete untruncated higher derivative theory Equation (71) does not propagate ghosts.

\section{A.4. Truncation to a Four Derivative Theory}

In the action Equation (71), the highest derivative terms come with $1 / \mu^{4}$. Dropping these results in a four-derivative action for $\phi$ with mass poles at $m^{2}=0$ and $m^{2}=2 \mu^{2} / 3$. On completing the squares, one can see that the massless mode is healthy, while the massive mode is now a ghost. Thus, the truncation has shifted the massive pole away from $2 \mu^{2}$ and turned it into a ghost. Of course, in this example, we know that the theory cannot be trusted near the massive pole, which is close to the truncation scale. Near the massive pole, higher derivative corrections become important, and including these both shifts the mass pole to its correct value, as well as eliminates the ghost.

The analogue of Equation (71) in the bimetric setup is given as an expansion in powers of derivatives, suppressed by the mass parameter. When truncated to four-derivative terms, the massive spin- 2 field becomes a ghost with a shifted mass, as an artifact of the truncation. The lesson is that the spin- 2 ghost in HD gravity must be understood in a similar way, although in the presence of interactions, the complete HD theory will not have a finite number of terms.

\section{A.5. Higher Derivative Treatment of Linearized Bimetric Theory}

Let us now repeat this procedure for spin-2 fluctuations on a curved background $\bar{g}_{\mu \nu}$. The notation here simplifies and streamlines the manipulations. Indices are raised using $\bar{g}$. Our starting point is the quadratic Fierz-Pauli action with linear interaction given by,

$S=\int \mathrm{d}^{d} x \sqrt{\bar{g}}\left[\frac{1}{2} h_{\mu \nu} D^{\mu \nu \rho \sigma}\left(\mu^{2}, \Lambda\right) h_{\rho \sigma}+\frac{1}{2} \tilde{h}_{\mu \nu} D^{\mu \nu \rho \sigma}\left(\tilde{\mu}^{2}, \tilde{\Lambda}\right) \tilde{h}_{\rho \sigma}+\lambda h_{\mu \nu} G_{(1)}^{\mu \nu \rho \sigma} \tilde{h}_{\rho \sigma}-h_{\mu \nu} T^{\mu \nu}-\tilde{h}_{\mu \nu} \tilde{T}^{\mu \nu}\right]$, 
where we have defined,

$$
D^{\mu \nu \rho \sigma}\left(\mu^{2}, \Lambda\right)=\mathcal{E}^{\mu \nu \rho \sigma}+\frac{\mu^{2}}{2} G_{(1)}^{\mu \nu \rho \sigma}-\frac{\Lambda}{d-2} G_{(1 / 2)}^{\mu \nu \rho \sigma}
$$

in terms of the linear Einstein operator $\mathcal{E}^{\mu \nu \rho \sigma}$ and the generalized DeWitt metrics, which we define through,

$$
G_{(\xi)}^{\mu \nu \rho \sigma}=\bar{g}^{\mu \nu} \bar{g}^{\rho \sigma}-\xi \bar{g}^{\mu(\rho} \bar{g}^{\sigma) \nu} .
$$

The field equations are given by,

$$
\begin{aligned}
& D^{\mu \nu \rho \sigma}\left(\mu^{2}, \Lambda\right) h_{\rho \sigma}+\lambda G_{(1)}^{\mu \nu \rho \sigma} \tilde{h}_{\rho \sigma}-T^{\mu \nu}=0, \\
& D^{\mu \nu \rho \sigma}\left(\tilde{\mu}^{2}, \tilde{\Lambda}\right) \tilde{h}_{\rho \sigma}+\lambda G_{(1)}^{\mu \nu \rho \sigma} h_{\rho \sigma}-\tilde{T}^{\mu \nu}=0,
\end{aligned}
$$

Restricting now to $\tilde{\Lambda}=\Lambda$ and $\tilde{\mu}^{2}=2 \lambda=\mu^{2}$, we can again simply add and subtract these equations to get,

$$
\begin{gathered}
D^{\mu \nu \rho \sigma}\left(2 \mu^{2}, \Lambda\right)\left(h_{\rho \sigma}+\tilde{h}_{\rho \sigma}\right)-\left(T^{\mu \nu}+\tilde{T}^{\mu \nu}\right)=0, \\
D^{\mu \nu \rho \sigma}(0, \Lambda)\left(h_{\rho \sigma}-\tilde{h}_{\rho \sigma}\right)-\left(T^{\mu \nu}-\tilde{T}^{\mu \nu}\right)=0 .
\end{gathered}
$$

These are the equations of a massive spin-2 field $h_{\mu \nu}^{+}=h_{\mu \nu}+\tilde{h}_{\mu \nu}$ with a mass $2 \mu^{2}$ and a massless spin-2 field $h_{\mu \nu}^{-}=h_{\mu \nu}-\tilde{h}_{\mu \nu}$ on a curved background. We can solve for $\tilde{h}_{\mu \nu}$ in Equation (79), to get,

$$
G_{(1)}^{\mu \nu \rho \sigma} \tilde{h}_{\rho \sigma}=-\frac{2}{\mu^{2}}\left[D^{\mu \nu \rho \sigma}\left(\mu^{2}, \Lambda\right) h_{\rho \sigma}-T^{\mu \nu}\right]
$$

and subsequently,

$$
\tilde{h}_{\mu \nu}=\frac{2}{\mu^{2}}\left[D_{\mu \nu}^{\rho \sigma}\left(\mu^{2}, \Lambda\right) h_{\rho \sigma}-T_{\mu \nu}-\frac{1}{d-1} \bar{g}_{\mu \nu}\left(D_{\alpha}^{\alpha}{ }^{\rho \sigma}\left(\mu^{2}, \Lambda\right) h_{\rho \sigma}-T\right)\right] .
$$

Inserting this solution in Equation (80) and using some basic manipulations (see the end of this section) of the DeWitt metrics, one obtains the higher derivative equation,

$$
D^{\mu \nu \alpha \beta}(0, \Lambda) G_{\alpha \beta \rho \sigma}^{(d-1)} D^{\rho \sigma \lambda \kappa}\left(2 \mu^{2}, \Lambda\right) h_{\lambda \kappa}-\left(D^{\mu \nu \alpha \beta}\left(\mu^{2}, \Lambda\right) G_{\alpha \beta \rho \sigma}^{(d-1)} T^{\rho \sigma}-\frac{d-1}{2} \mu^{2} \tilde{T}^{\mu \nu}\right)=0 .
$$

Again, the structure of the higher derivative operator hitting $h_{\lambda \kappa}$ is the composite of a zero mass operator and a massive operator for a mass $2 \mu^{2}$. The contraction of these operators is of course a bit more complex now, involving a DeWitt metric. In fact, this is the inverse of $G_{(1)}$, in the sense that,

$$
G_{(1)}^{\mu \nu \rho \sigma} G_{\alpha \beta \rho \sigma}^{(d-1)}=\frac{d-1}{2}\left(\delta_{\alpha}^{\mu} \delta_{\beta}^{\nu}+\delta_{\beta}^{\mu} \delta_{\alpha}^{\nu}\right)
$$

In the source contribution of Equation (85), one of the sources is contracted with an operator $\sim$ $\delta \tilde{h}^{\mu \nu} / \delta h_{\rho \sigma}$. All of this is in complete analogy to the scalar field example and generalizes that discussion in a very straightforward way, the only complication being the metric of contraction. The insertion of the solution Equations (83) and (84) into the action also proceeds in an analogue fashion and again results in an operator $D\left(\mu^{2}, \Lambda\right)$ contracting Equation (85) with the DeWitt metric $G^{(d-1)}$. This makes it obvious 
that the implications of absence of a ghost for the untruncated theory go through just as in the scalar field example, and we do not provide the details of this here.

In order to check the details at the level of the action, it is useful to observe that,

$$
G_{(1)}^{\mu \nu \alpha \beta} G_{(1) \alpha \beta}{ }^{\rho \sigma}+G_{(1)}^{\mu \nu \rho \sigma}-\frac{1}{d-1} G_{(1) \alpha}^{\mu \nu \alpha} G_{(1) \beta}^{\beta}{ }^{\rho \sigma}=0,
$$

and,

$$
G_{(1) \alpha}^{\alpha \rho \sigma}=G_{(1) \alpha}^{\rho \sigma \alpha}=(d-1) \bar{g}^{\rho \sigma}
$$

together with (for any $D$ ),

$$
D^{\alpha \beta \rho \sigma} G_{(1) \alpha \beta}^{\mu \nu}=D_{\alpha}^{\alpha \rho \sigma} \bar{g}^{\mu \nu}-D^{\mu \nu \rho \sigma}, \quad D_{\alpha \beta}^{\mu \nu} G_{(1)}^{\alpha \beta \rho \sigma}=D_{\alpha}^{\mu \nu \alpha} \bar{g}^{\rho \sigma}-D^{\mu \nu \rho \sigma} .
$$

These results can be used to show that,

$$
\begin{aligned}
& D^{\mu \nu \alpha \beta}\left(\mu^{2}, \Lambda\right) D_{\alpha \beta}{ }^{\rho \sigma}\left(\mu^{2}, \Lambda\right)-\frac{1}{d-1} D_{\alpha}^{\mu \nu \alpha}\left(\mu^{2}, \Lambda\right) D_{\beta}^{\beta}{ }^{\rho \sigma}\left(\mu^{2}, \Lambda\right)+\frac{\mu^{4}}{4} G_{(1)}^{\mu \nu \rho \sigma} \\
& =D^{\mu \nu \alpha \beta}(0, \Lambda) D_{\alpha \beta}{ }^{\rho \sigma}\left(2 \mu^{2}, \Lambda\right)-\frac{1}{d-1} D_{\alpha}^{\mu \nu \alpha}(0, \Lambda) D_{\beta}^{\beta}{ }_{\beta}{ }^{\rho \sigma}\left(2 \mu^{2}, \Lambda\right) .
\end{aligned}
$$

Together with the solutions Equations (83) and (84), these relations make the manipulations straightforward.

\section{B. The General Perturbative Solution of the $g_{\mu \nu}$ Equation for $f_{\mu \nu}$}

For general $\beta_{n}$, finding an exact solution for $S=\sqrt{g^{-1} f}$ is not feasible. (In principle, exact solutions can be obtained implicitly by considering higher powers of the $g_{\mu \nu}$-equation when written in the form $R_{\nu}^{\mu}=\sum_{r} q_{r}\left(S^{r}\right)_{\nu}^{\mu}$, where $q_{r}$ are functions of $\beta_{n}$ and $e_{n}(S)$. On tracing and expressing $\operatorname{Tr}\left(S^{r}\right)$ for $r>d$ in terms $\operatorname{Tr}\left(S^{n}\right)$ with $n \leq d$, one can obtain enough equations to determine all $e_{n}(S)$. Then, the $g$-equation can be used to determine $S$ in terms of the curvatures.) However, one can find a perturbative solution in powers of $\frac{1}{m^{2}}$. The equation of motion for $g_{\mu \nu}$ in terms of its Schouten tensor $P_{\mu \nu}$ becomes,

$$
\operatorname{Tr} P \delta_{\nu}^{\mu}-P_{\nu}^{\mu}=m^{2} \sum_{n=0}^{d-1}(-1)^{n} \beta_{n} \mathbb{Y}_{(n) \nu}^{\mu}(S),
$$

where the matrices $\mathbb{Y}_{(n)}(S)$ are defined in Equation (17), and here and in the following, we raise and lower indices with $g_{\mu \nu}$. Our goal is to solve the Equation (91) for $S_{\nu}^{\mu}$ perturbatively in $P_{\nu}^{\mu} / m^{2}$. Since the solution for $S_{\nu}^{\mu}$ is a polynomial in $P_{\nu}^{\mu}$, the resulting action for $g_{\mu \nu}$ will be a higher curvature theory with coefficients given in terms of the parameters of the bimetric model. In order to find the perturbative solution for $S_{\nu}^{\mu}$, consider the general ansatz,

$$
\begin{aligned}
S_{\nu}^{\mu}=a \delta_{\nu}^{\mu} & +\frac{1}{m^{2}}\left(b_{1} P_{\nu}^{\mu}+b_{2} \operatorname{Tr} P \delta_{\nu}^{\mu}\right) \\
& +\frac{1}{m^{4}}\left(c_{1} P_{\nu}^{\mu 2}+c_{2} P_{\nu}^{\mu} \operatorname{Tr} P+c_{3} \operatorname{Tr}\left(P^{2}\right) \delta_{\nu}^{\mu}+c_{4}(\operatorname{Tr} P)^{2} \delta_{\nu}^{\mu}\right)+\mathcal{O}\left(m^{-6}\right) .
\end{aligned}
$$

When we plug this into Equation (91), the coefficients of different terms $\delta_{\nu}^{\mu}, P_{\nu}^{\mu}, \delta_{\nu}^{\mu} \operatorname{Tr} P, P_{\nu}^{\mu 2}, \ldots$ have to vanish separately. This will determine all of the coefficients $a, b_{i}, c_{i}$ in the ansatz for $S_{\nu}^{\mu}$. 
To simplify the calculation, define $M_{\nu}^{\mu} \equiv S_{\nu}^{\mu} / a-\delta_{\nu}^{\mu}$ or, $S_{\nu}^{\mu}=a\left(\delta_{\nu}^{\mu}+M_{\nu}^{\mu}\right)$. Then, using,

$$
e_{k}(S)=a^{k} \sum_{m=0}^{k}\left(\begin{array}{l}
d-m \\
k-m
\end{array}\right) e_{m}(M), \quad\left(S^{n-k}\right)_{\nu}^{\mu}=a^{n-k} \sum_{r=0}^{n-k}\left(\begin{array}{c}
n-k \\
r
\end{array}\right)\left(M^{r}\right)_{\nu}^{\mu}
$$

the equations of motion can be written as,

$$
\operatorname{Tr} P \delta_{\nu}^{\mu}-P_{\nu}^{\mu}=m^{2} \sum_{n=0}^{d-1} \beta_{n} a^{n} \sum_{k=0}^{n} \sum_{m=0}^{k} \sum_{r=0}^{n-k}(-1)^{n+k}\left(\begin{array}{c}
d-m \\
k-m
\end{array}\right)\left(\begin{array}{c}
n-k \\
r
\end{array}\right) e_{m}(M)\left(M^{r}\right)^{\mu}{ }_{\nu} .
$$

Since $M_{\nu}^{\mu}$ starts with $m^{-2}$, a power of $M^{l}$ will contribute to orders $m^{-2 l}$ and higher. In the following, we work out the first three orders explicitly. To simplify the notation, we use the sums $s_{k}$ defined in Equation (24) that will be frequently encountered below.

Zeroth order: The equation of motion at $\mathcal{O}\left(\mathrm{m}^{0}\right)$ is obtained from terms with the last two sums in Equation (94) restricted to $(r, m)=(0,0)$. This gives $s_{0} \equiv \sum_{n=0}^{d-1}\left(\begin{array}{c}d-1 \\ n\end{array}\right) \beta_{n} a^{n}=0$, Equation (25). This is a polynomial equation in $a$ that determines it in terms of the $\beta_{n}$.

First order: At $\mathcal{O}\left(m^{-2}\right)$, terms with $(r, m)=(1,0)$ and $(r, m)=(0,1)$ contribute in Equation (94). Using simple identities for the binomial coefficients, we obtain for this order,

$$
\operatorname{Tr} P \delta_{\nu}^{\mu}-P_{\nu}^{\mu}=\frac{1}{a}\left[\left((d-1) b_{2}+b_{1}\right) \operatorname{Tr} P \delta_{\nu}^{\mu}-b_{1} P_{\nu}^{\mu}\right] s_{1},
$$

where $s_{1}$ is defined in Equation (24). Comparing the coefficients of $\operatorname{Tr} P \delta_{\nu}^{\mu}$ and $P_{\nu}^{\mu}$ separately, we find the following equation that determines $b_{1}$ and $b_{2}$ in terms of the $\beta_{n}$.

$$
b_{2}=0, \quad b_{1}=\frac{a}{s_{1}} .
$$

Second order: At order $\mathcal{O}\left(m^{-4}\right)$, we can have contributions from the following combinations of $(r, m)$,

$$
(1,0), \quad(2,0), \quad(1,1), \quad(0,1), \quad(0,2) .
$$

Demanding the coefficients of the different terms at this order to vanish separately then gives the following system of equations for the coefficients $c_{i}$ in the ansatz Equation (92),

$$
\begin{gathered}
s_{1} c_{1}-\frac{b_{1}^{2} s_{2}}{a}=0, \quad s_{1} c_{2}+\frac{b_{1}^{2} s_{2}}{a}=0, \\
s_{1}\left(c_{1}+d c_{3}\right)-s_{1} c_{3}-\frac{b_{1}^{2} s_{2}}{2 a}=0, \quad s_{1}\left(c_{2}+d c_{4}\right)-s_{1} c_{4}+\frac{b_{1}^{2} s_{2}}{2 a}=0 .
\end{gathered}
$$

The solution is easily found to be,

$$
c_{1}=-c_{2}=\frac{b_{1}^{2} s_{2}}{a s_{1}}=\frac{a s_{2}}{s_{1}^{3}}, \quad c_{3}=-c_{4}=\frac{c_{2}}{2(d-1)}=-\frac{a s_{2}}{2(d-1) s_{1}^{3}},
$$

where we have also used the solution for $b_{1}$ given in Equation (26). Putting everything together, the solution for $S^{\mu}{ }_{\nu}$ to $\mathcal{O}\left(\mathrm{m}^{-4}\right)$ becomes Equation (28). The procedure can straightforwardly be continued to compute the solution for $S_{\nu}^{\mu}$ to any order in $m^{-2}$. 


\section{References}

1. Hassan, S.F.; Rosen, R.A. Bimetric Gravity from Ghost-free Massive Gravity. J. High Energy Phys. 2012, 2012, 126.

2. Hassan, S.F.; Rosen, R.A. Confirmation of the Secondary Constraint and Absence of Ghost in Massive Gravity and Bimetric Gravity. J. High Energy Phys. 2012, 2012, 123.

3. Hassan, S.F.; Schmidt-May, A.; von Strauss, M. On Partially Massless Bimetric Gravity. Phys. Lett. B 2012, 726, 834-838.

4. Hassan, S.F.; Schmidt-May, A.; von Strauss, M. Bimetric Theory and Partial Masslessness with Lanczos-Lovelock Terms in Arbitrary Dimensions. Class. Quant. Grav. 2012, 30, 184010

5. Stelle, K.S. Renormalization of Higher Derivative Quantum Gravity. Phys. Rev. D 1977, 16, 953-969.

6. Stelle, K.S. Classical Gravity with Higher Derivatives. Gen. Rel. Grav. 1978, 9, 353-371.

7. Bergshoeff, E.A.; Hohm, O.; Townsend, P.K. Massive Gravity in Three Dimensions. Phys. Rev. Lett. 2009, 102, 201301.

8. Ohta, N. A Complete Classification of Higher Derivative Gravity in 3D and Criticality in 4D. Class. Quant. Grav. 2012, 29, 015002.

9. Kleinschmidt, A.; Nutma, T.; Virmani, A. On unitary subsectors of polycritical gravities. Gen. Rel. Grav. 2013, 45, 727-749.

10. Eliezer, D.A.; Woodard, R.P. The Problem of Nonlocality in String Theory. Nucl. Phys. B 1989, 325, 389-469.

11. Simon, J.Z. Higher Derivative Lagrangians, Nonlocality, Problems And Solutions. Phys. Rev. D 1990, 41, 3720-3733.

12. Biswas, T.; Mazumdar, A.; Siegel, W. Bouncing universes in string-inspired gravity. J. Cosmol. Astropart. Phys. 2006, doi:10.1088/1475-7516/2006/03/009.

13. Biswas, T.; Gerwick, E.; Koivisto, T.; Mazumdar, A. Towards singularity and ghost free theories of gravity. Phys. Rev. Lett. 2012, 108, 031101.

14. Biswas, T.; Koshelev, A.S.; Mazumdar, A.; Vernov, S.Y. Stable bounce and inflation in non-local higher derivative cosmology. J. Cosmol. Astropart. Phys. 2012, doi:10.1088/1475-7516/ 2012/08/024.

15. Nojiri, S.I.; Odintsov, S.D. Ghost-free $F(R)$ bigravity and accelerating cosmology. Phys. Lett. $B$ 2012, doi:10.1016/j.physletb.2012.08.049.

16. Bach, R. Zur Weylschen Relativitätstheorie und der Weylschen Erweiterung des Krümmungsbegriffs. Math. Zeitschr. 1921, 9, 110-135. (In German)

17. Kaku, M.; Townsend, P.K.; van Nieuwenhuizen, P. Gauge Theory of the Conformal and Superconformal Group. Phys. Lett. B 1977, 69, 304-308.

18. Fradkin, E.S.; Tseytlin, A.A. Renormalizable asymptotically free quantum theory of gravity. Nucl. Phys. B 1982, 201, 469-491.

19. Lee, S.C.; van Nieuwenhuizen, P. Counting of States In Higher Derivative Field Theories. Phys. Rev. D 1982, 26, 934-937. 
20. Riegert, R.J. The Particle Content Of Linearized Conformal Gravity. Phys. Lett. A 1984, 105, 110-112.

21. Maldacena, J. Einstein Gravity from Conformal Gravity. 2011, arXiv:1105.5632 [hep-th].

22. Lu, H.; Pang, Y.; Pope, C.N. Conformal Gravity and Extensions of Critical Gravity. Phys. Rev. D 2011, 84, 064001.

23. Lu, H.; Pang, Y.; Pope, C.N. Black Holes in Six-dimensional Conformal Gravity. Phys. Rev. D 2013, 87, 104013.

24. Metsaev, R.R. Ordinary-derivative formulation of conformal totally symmetric arbitrary spin bosonic fields. J. High Energy Phys. 2012, 2012, 062.

25. Mannheim, P.D. Making the Case for Conformal Gravity. Found. Phys. 2012, 42, 388-420.

26. Schmidt, H.J. Fourth order gravity: Equations, history, and applications to cosmology. Int. J. Geom. Meth. Mod. Phys. 2007, 4, 209-248.

27. Alexandrov, S.; Krasnov, K.; Speziale, S. Chiral description of ghost-free massive gravity. J. High Energy Phys. 2013, 2013, 068.

28. Soloviev, V.O.; Tchichikina, M.V. Bigravity in Kuchar's Hamiltonian formalism: The special case. Phys. Rev. D 2013, 88, 084026.

29. Hassan, S.F.; Schmidt-May, A.; von Strauss, M. On Consistent Theories of Massive Spin-2 Fields Coupled to Gravity. J. High Energy Phys. 2013, 2013, 86.

30. Volkov, M.S. Cosmological solutions with massive gravitons in the bigravity theory. J. High Energy Phys. 2012, 2012, 035.

31. Von Strauss, M.; Schmidt-May, A.; Enander, J.; Mortsell, E.; Hassan, S.F. Cosmological Solutions in Bimetric Gravity and their Observational Tests. J. Cosmol. Astropart. Phys. 2012, doi:10.1088/1475-7516/2012/03/042.

32. Comelli, D.; Crisostomi, M.; Nesti, F; Pilo, L. FRW Cosmology in ghost-free Massive Gravity. J. High Energy Phys. 2012, doi:10.1007/JHEP03(2012)067.

33. Berg, M.; Buchberger, I.; Enander, J.; Mortsell, E.; Sjors, S. Growth Histories in Bimetric Massive Gravity. J. Cosmol. Astropart. Phys. 2012, doi:10.1088/1475-7516/2012/12/021.

34. Park, M.; Sorbo, L. Vacua and instantons of ghost-free massive gravity. Phys. Rev. D 2013, 87, 024041.

35. Sakakihara, Y.; Soda, J.; Takahashi, T. On Cosmic No-hair in Bimetric Gravity and the Higuchi Bound. Prog. Theor. Exp. Phys. 2013, 2013, $033 \mathrm{E} 02$.

36. Akrami, Y.; Koivisto, T.S.; Sandstad, M. Accelerated expansion from ghost-free bigravity: a statistical analysis with improved generality. J. High Energy Phys. 2013, 2013, 99.

37. Capozziello, S.; Martin-Moruno, P. Bounces, turnarounds and singularities in bimetric gravity. Phys. Lett. B 2013, 719, 14-17.

38. Mohseni, M. Gravitational Waves in Ghost Free Bimetric Gravity. J. Cosmol. Astropart. Phys. 2012, doi:10.1088/1475-7516/2012/11/023.

39. Baccetti, V.; Martin-Moruno, P.; Visser, M. Gordon and Kerr-Schild ansatze in massive and bimetric gravity. J. High Energy Phys. 2012, doi:10.1007/JHEP08(2012)108.

40. Baccetti, V.; Martin-Moruno, P.; Visser, M. Null Energy Condition violations in bimetric gravity. J. High Energy Phys. 2012, doi:10.1007/JHEP08(2012)148. 
41. Baccetti, V.; Martin-Moruno, P.; Visser, M. Massive gravity from bimetric gravity. Class. Quant. Grav. 2013, 30, 015004.

42. Volkov, M.S. Hairy black holes in the ghost-free bigravity theory. Phys. Rev. D 2012, 85, 124043.

43. Myrzakulov, R.; Shahalam, M. Statefinder hierarchy of bimetric and galileon models for concordance cosmology. J. Cosmol. Astropart. Phys. 2013, 10, 047.

44. Maeda, K.-I.; Volkov, M.S. Anisotropic universes in the ghost-free bigravity. Phys. Rev. D 2013, 87, 104009.

45. De Rham, C.; Gabadadze, G. Generalization of the Fierz-Pauli Action. Phys. Rev. D 2010, $82,044020$.

46. De Rham, C.; Gabadadze, G.; Tolley, A.J. Resummation of Massive Gravity. Phys. Rev. Lett. 2011, 106, 231101.

47. De Rham, C. Massive Gravity. Living Rev. Rel. 2014, doi:10.12942/lrr-2014-7.

48. Hassan, S.F.; Rosen, R.A. Resolving the Ghost Problem in nonlinear Massive Gravity. Phys. Rev. Lett. 2012, 108, 041101.

49. Hassan, S.F.; Rosen, R.A.; Schmidt-May, A. Ghost-free Massive Gravity with a General Reference Metric. J. High Energy Phys. 2012, doi:10.1007/JHEP02(2012)026.

50. Hassan, S.F.; Schmidt-May, A.; von Strauss, M. Particular Solutions in Bimetric Theory and Their Implications. Int. J. Mod. Phys. D 2014, 23, 1443002.

51. Akrami, Y.; Hassan, S.F.; Könnig, F.; Schmidt-May, A.; Solomon, A.R. Bimetric gravity is cosmologically viable. Phys. Lett. B 2015, 748, 37-44.

52. Deser, S.; Waldron, A. Acausality of Massive Gravity. Phys. Rev. Lett. 2013, 110, 111101.

53. Deser, S.; Sandora, M.; Waldron, A.; Zahariade, G. Covariant constraints for generic massive gravity and analysis of its characteristics. Phys. Rev. D 2014, 90, 104043.

54. Deser, S.; Sandora, M.; Waldron, A. Nonlinear Partially Massless from Massive Gravity? Phys. Rev. D 2013, 87, 101501.

55. De Rham, C.; Hinterbichler, K.; Rosen, R.A.; Tolley, A.J. Evidence for and Obstructions to Non-Linear Partially Massless Gravity. Phys. Rev. D 2013, 88, 024003.

56. Higuchi, A. Forbidden Mass Range For Spin-2 Field Theory In De Sitter Space-time. Nucl. Phys. B 1987, 282, 397-436.

57. Deser, S.; Waldron, A. Partial masslessness of higher spins in (A)dS. Nucl. Phys. B 2001, 607, 577-604.

58. Francia, D.; Mourad, J.; Sagnotti, A. (A)dS exchanges and partially-massless higher spins. Nucl. Phys. B 2008, 804, 383-420.

59. Joung, E.; Lopez, L.; Taronna, M. On the cubic interactions of massive and partially-massless higher spins in (A)dS. J. High Energy Phys. 2012, doi:10.1007/JHEP07(2012)041.

60. Joung, E.; Lopez, L.; Taronna, M. Generating functions of (partially-)massless higher-spin cubic interactions. J. High Energy Phys. 2013, doi:10.1007/JHEP01(2013)168.

61. Zinoviev, Y.M. All spin-2 cubic vertices with two derivatives. Nucl. Phys. B 2013, 872, 21-37.

62. Zinoviev, Y.M. On massive spin 2 interactions. Nucl. Phys. B 2007, 770, 83-106.

63. Deser, S.; Joung, E.; Waldron, A. Gravitational and self-coupling of partially massless spin 2. Phys. Rev. D 2012, 86, 104004. 
64. Boulware, D.G.; Deser, S. Can gravitation have a finite range? Phys. Rev. D 1972, 6, 3368-3382.

65. De Felice, A.; Gumrukcuoglu, A.E.; Lin, C.; Mukohyama, S. Nonlinear stability of cosmological solutions in massive gravity.

J. Cosmol. Astropart. Phys.

2013, doi:10.1088/1475-7516/2013/05/035.

66. Hassan, S.F.; Rosen, R.A. On nonlinear Actions for Massive Gravity. J. High Energy Phys. 2011, doi:10.1007/JHEP07(2011)009.

67. Hassan, S.F.; Schmidt-May, A.; von Strauss, M. Proof of Consistency of Nonlinear Massive Gravity in the Stúckelberg Formulation. Phys. Lett. B 2012, 715, 335-339.

68. De Rham, C.; Renaux-Petel, S. Massive Gravity on de Sitter and Unique Candidate for Partially Massless Gravity. J. Cosmol. Astropart. Phys. 2013, doi:10.1088/1475-7516/2013/01/035.

69. Paulos, M.F.; Tolley, A.J. Massive Gravity Theories and limits of Ghost-free Bigravity models. J. High Energy Phys. 2012, doi:10.1007/JHEP09(2012)002.

70. Volkov, M.S. Exact self-accelerating cosmologies in the ghost-free massive gravity-The detailed derivation. Phys. Rev. D 2012, 86, 104022.

71. Wald, R.M. General Relativity; University of Chicago press: Chicago, IL, USA, 1984; p. 491

72. Nurowski, P.; Plebanski, J.F. Nonvacuum twisting type N metrics. Class. Quant. Grav. 2001, 18, 341-351.

73. Liu, H.-S.; Lu, H.; Pope, C.N.; Vazquez-Poritz, J. Not Conformally-Einstein Metrics in Conformal Gravity. Class. Quant. Grav. 2013, 30, 165015.

74. Deffayet, C.; Mourad, J.; Zahariade, G. A note on "symmetric" vielbeins in bimetric, massive, perturbative and non perturbative gravities. J. High Energy Phys. 2013, doi:10.1007/JHEP03(2013)086.

75. Hassan, S.F.; Kocic, M.; Schmidt-May, A. Absence of ghost in a new bimetric-matter coupling. 2014, arXiv:1409.1909[hep-th].

76. Hinterbichler, K.; Rosen, R.A. Interacting Spin-2 Fields. J. High Energy Phys. 2012 , doi:10.1007/JHEP07(2012)047.

77. Hassan, S.F.; Schmidt-May, A.; von Strauss, M. Metric Formulation of Ghost-Free Multivielbein Theory. 2012, arXiv:1204.5202 [hep-th].

78. Bonora, L.; Pasti, P.; Bregola, M. Weyl Cocycles. Class. Quant. Grav. 1986, doi:10.1088/0264-9381/3/4/018.

79. Metsaev, R.R. 6d conformal gravity. J. Phys. A 2011, 44, 175402.

80. Boulanger, N.; Erdmenger, J. A Classification of local Weyl invariants in D=8. Class. Quant. Grav. 2004, 21, 4305-4316.

81. Deser, S.; Sandora, M.; Waldron, A. No consistent bimetric gravity? Phys. Rev. D 2013, $88,081501$.

82. Joung, E.; Li, W.; Taronna, M. No-Go Theorems for Unitary and Interacting Partially Massless Spin-Two Fields. Phys. Rev. Lett. 2014, 113, 091101.

83. Garcia-Saenz, S.; Rosen, R.A. A non-linear extension of the spin-2 partially massless symmetry. J. High Energy Phys. 2015, doi:10.1007/JHEP05(2015)042. 
84. Hassan, S.F.; Schmidt-May, A.; von Strauss, M. Extended Weyl Invariance in a Bimetric Model. 2015, In preparation.

(c) 2015 by the authors; licensee MDPI, Basel, Switzerland. This article is an open access article distributed under the terms and conditions of the Creative Commons Attribution license (http://creativecommons.org/licenses/by/4.0/). 